Southern Illinois University Carbondale

OpenSIUC

Articles and Preprints

Department of Mathematics

6-19-2010

\title{
State and Feedback Linearizations of Single-Input Control Systems
}

Issa Amadou Tall

Southern Illinois University Carbondale, itall@math.siu.edu

Follow this and additional works at: http://opensiuc.lib.siu.edu/math_articles Published in Tall, I. A. (2010). State and feedback linearizations of single-input control systems. Systems \& Control Letters, 59(7), 449-451. doi: 10.1016/j.sysconle.2010.05.006

\section{Recommended Citation}

Tall, Issa A. "State and Feedback Linearizations of Single-Input Control Systems." (Jun 2010).

This Article is brought to you for free and open access by the Department of Mathematics at OpenSIUC. It has been accepted for inclusion in Articles and Preprints by an authorized administrator of OpenSIUC. For more information, please contact opensiuc@lib.siu.edu. 
State and Feedback Linearizations of Single-Input Control Systems

Issa Amadou Tall

Southern Illinois University Carbondale,MC 4408, 1245 Lincoln Drive, Carbondale IL, 62901, USA, itall@math.siu.edu. 


\title{
State and Feedback Linearizations of Single-Input Control Systems
}

\author{
Issa Amadou Tall \\ Southern Illinois University Carbondale,MC 4408, 1245 Lincoln Drive, Carbondale IL, 62901, USA, itall@math.siu.edu.
}

\begin{abstract}
In this paper we address the problem of state (resp. feedback) linearization of nonlinear single-input control systems using state (resp. feedback) coordinate transformations. Although necessary and sufficient geometric conditions have been provided in the early eighties, the problems of finding the state (resp. feedback) linearizing coordinates are subject to solving systems of partial differential equations. We will provide here a solution to the two problems by defining algorithms allowing to compute explicitly the linearizing state (resp. feedback) coordinates for any nonlinear control system that is indeed linearizable (resp. feedback linearizable). Each algorithm is performed using a maximum of $n-1$ steps ( $n$ being the dimension of the system) and they are made possible by explicitly solving the Flow-box or straightening theorem. We illustrate with several examples borrowed from the literature.
\end{abstract}

\section{Introduction and Preliminaries}

In the late seventies and early eighties the problem of transforming a nonlinear control system, via change of coordinates and feedback, into a linear one, has been introduced and is known today as feedback linearization. The feedback classification was applied first to linear systems for which a complete picture has been made possible. The controllability, observability, reachability, and realization of linear systems have been expressed in very simple algebraic terms. A crucial property of linear controllable systems is that they can be stabilized by linear feedback controllers. Because of the simplicity of their analysis and design; because several physical systems can be modeled using linear dynamics, and due to the observation that some nonlinear phenomena are just hidden linear systems, it is thus not surprising that the linearization problems were (and still are) of paramount importance and have attracted much attention. Uncovering the hidden linear properties of nonlinear control systems turns out to be useful in analyzing the latter systems though some global properties might be lost during the operation. This paper proposes a way of finding the linearizing coordinates. To give a brief description of the linearization problems we will start first by recalling some basic facts about linear systems.

\subsection{Linear Systems}

We consider linear systems of the form

$$
\Lambda:\left\{\begin{array}{l}
\dot{x}=F x+G u=F x+\sum_{i=1}^{m} G_{i} u_{i}, \\
y=H x
\end{array}\right.
$$

where $x \in \mathbb{R}^{n}, F x$ and $G_{1}, \ldots, G_{m}$ are, respectively, linear and constant vector fields on $\mathbb{R}^{n}, H x$ a linear vector field on $\mathbb{R}^{p}$, and $u=\left(u_{1}, \ldots, u_{m}\right)^{\top} \in \mathbb{R}^{m}$. To any linear system $\Lambda$ we attach two geometric objects: (a) the controllability space

$$
\mathcal{C}_{n}=\operatorname{span}\left[G F G \cdots F^{n-1} G\right]
$$

as a $n \times(n m)$ matrix whose columns are those of the matrices $F^{i-1} G, i=1, \ldots, n$, and (b) the observability space

$$
\mathcal{O}_{n}=\operatorname{span}\left[H^{\mathrm{T}}(H F)^{\mathrm{T}} \cdots\left(H F^{n-1}\right)^{\mathrm{T}}\right]^{\mathrm{T}},
$$

as a $(n p) \times n$ matrix whose rows are those of the matrices $H F^{i-1}, i=1, \ldots, n$. The system $\Lambda$ is controllable (resp. observable) if and only if $\operatorname{dim} \mathcal{C}_{n}=n\left(\operatorname{resp} . \operatorname{rank} \mathcal{O}_{n}=n\right)$. By a linear change of coordinates $\tilde{x}=T x$ and a linear feedback $u=K x+L v$, where $T, K$, and $L$ are matrices of appropriate sizes, $T$ and $L$ being invertible, the system $\Lambda$ is transformed into a linear equivalent one

$$
\tilde{\Lambda}:\left\{\begin{array}{l}
\dot{\tilde{x}}=\tilde{F} \tilde{x}+\tilde{G} v \\
\tilde{y}=\tilde{H} \tilde{x}
\end{array}\right.
$$

with $\tilde{F} \tilde{x}=T(F+G K) T^{-1}, \tilde{G}=T G L$ and $\tilde{H}=H T^{-1}$. It is shown in the literature [2], [14] that the dimension of $\mathcal{C}_{n}$ and the rank of $\mathcal{O}_{n}$, (hence the controllability and observability), are two invariants of the feedback classification of linear systems. The problem of feedback classification for linear systems $\Lambda$ is to find linear state coordinates $w=T x$ and linear feedback $u=K x+L v$ that map $\Lambda$ into a simpler linear system $\tilde{\Lambda}$. It is a classical result of the linear control theory (see, e.g., [2], [14]) that any linear controllable system is feedback equivalent to the following Brunovský canonical form (single-input case):

$$
\Lambda_{B r}: \dot{w}=A w+b v, w \in \mathbb{R}^{n}, v \in \mathbb{R}
$$

April 28, 2010 
where

$$
A=\left(\begin{array}{ccccc}
0 & 1 & 0 & \cdots & 0 \\
0 & 0 & 1 & \cdots & 0 \\
\vdots & \vdots & \vdots & \ddots & \vdots \\
0 & \cdots & 0 & \cdots & 1 \\
0 & 0 & 0 & \cdots & 0
\end{array}\right) \quad b=\left(\begin{array}{c}
0 \\
0 \\
\vdots \\
0 \\
1
\end{array}\right)
$$

When $K=0$ and $L=1$, that is, only a linear change of coordinates is applied, the system $\Lambda_{B r}$ is replaced by $\Lambda_{\lambda}: \dot{w}=A_{\lambda} w+b v, w \in \mathbb{R}^{n}, v \in \mathbb{R}$, where $A_{\lambda}$ is the matrix $A$ with the first column replaced by $\lambda=\left(\lambda_{1}, \ldots, \lambda_{n}\right)^{\mathrm{T}}$. In the case of multi-input linear control systems, we can find positive integers $\rho_{1} \geq \cdots \geq \rho_{m}, \sum_{i=1}^{m} \rho_{i}=n$ (called controllability, Brunovský or Kronecker indices) such that $\Lambda_{B r}$ is a cascade of single-input linear systems $\Lambda_{B r}^{1}, \ldots, \Lambda_{B r}^{m}$ :

$$
\Lambda_{B r}^{i}: \dot{w}_{i}=A_{i} w_{i}+b_{i} v_{i}, w_{i} \in \mathbb{R}^{\rho_{i}}, v_{i} \in \mathbb{R},
$$

with $A=\operatorname{diag}\left\{A_{1}, \ldots, A_{m}\right\}$ and $b=\operatorname{diag}\left\{b_{1}, \ldots, b_{m}\right\}$. For a complete description and geometric interpretation of the Brunovský controllability indices we refer to the literature [2], [11], [12] , [13], [14], [25] and references therein.

\subsection{Nonlinear Systems and Linearization Problems.}

Consider a smooth (resp. analytic) control-affine system

$$
\Sigma: \dot{x}=f(x)+g(x) u=f(x)+\sum_{i=1}^{m} g_{i}(x) u_{i}, x \in \mathbb{R}^{n}
$$

around an equilibrium $\left(x_{e}, u_{e}\right)$, that is, $f\left(x_{e}\right)+g\left(x_{e}\right) u_{e}=$ 0 . We assume that $f, g_{1}, \ldots, g_{m}$ are smooth (resp. analytic) and $\left(x_{e}, u_{e}\right)=(0,0) \in \mathbb{R}^{n} \times \mathbb{R}^{m}$ or simply $f(0)=0$. Let

$$
\tilde{\Sigma}: \dot{\tilde{x}}=\tilde{f}(\tilde{x})+\tilde{g}(\tilde{x}) v=\tilde{f}(\tilde{x})+\sum_{i=1}^{m} \tilde{g}_{i}(\tilde{x}) v_{i}, \tilde{x} \in \mathbb{R}^{n}
$$

be another smooth (resp. analytic) control-affine system. The systems $\Sigma$ and $\tilde{\Sigma}$ are called feedback equivalent if there exist

$$
\Gamma:\left\{\begin{aligned}
\tilde{x} & =\phi(x) \\
u & =\alpha(x)+\beta(x) v
\end{aligned}\right.
$$

a transformation that maps $\Sigma$ into $\tilde{\Sigma}$, that is, such that

$$
(P D E s)\left\{\begin{aligned}
\mathrm{d} \phi(x) \cdot(f(x)+g(x) \alpha(x)) & =\tilde{f}(\phi(x)) \\
\mathrm{d} \phi(x) \cdot(g(x) \beta(x)) & =\tilde{g}(\phi(x)) .
\end{aligned}\right.
$$

We will briefly write $\Gamma=(\phi, \alpha, \beta)$ and put $\Gamma_{*} \Sigma=\tilde{\Sigma}$. When $\alpha \equiv 0$ and $\beta \equiv \operatorname{id}_{\mathrm{m}}$, then we say that $\Sigma$ and $\tilde{\Sigma}$ are state equivalent, and we simply write $\phi_{*} \Sigma=\tilde{\Sigma}$. The following two problems were considered in the late 1970s by Brockett [4], and Krener [16].
Problem 1. When does there exist a local diffeomorphism $w=\phi(x)$ defining new coordinates $w=\left(w_{1}, \ldots, w_{n}\right)^{\mathrm{T}}$ in which the transformed system $\phi_{*} \Sigma$ takes the linear form

$$
\Lambda: \dot{w}=F w+G u=F w+\sum_{i=1}^{m} G_{i} u_{i}, w \in \mathbb{R}^{n}, u \in \mathbb{R}^{m} ?
$$

Problem 2. When did there exist a (local) feedback transformation $\Gamma=(\phi, \alpha, \beta)$ that takes $\Sigma$ into a linear system

$$
\Lambda: \dot{w}=A w+B v=A w+\sum_{i=1}^{m} b_{i} v_{i}, w \in \mathbb{R}^{n}, v \in \mathbb{R}^{m} ?
$$

When Problem 1 (resp. Problem 2) is solvable, then the system $\Sigma$ is called state linearizable, shortly $\mathcal{S}$-linearizable (resp. feedback linearizable, shortly, $\mathcal{F}$ linearizable). Problem $\mathbf{1}$ was completely solved by Krener [16] and Problem 2 partially by Brockett [4] for $m=1$ and $\beta$ constant. A generalization was obtained independently by Hunt and $\mathrm{Su}$ [11], Jakubczyk and Respondek [13], who gave necessary and sufficient geometric conditions in terms of Lie brackets of vector fields defining the system. Indeed, attach to $\Sigma$ the sequence of nested distributions $\mathcal{D}^{1} \subset \mathcal{D}^{2} \subset \cdots \subset \mathcal{D}^{n}$, where

$$
\mathcal{D}^{k}=\left\{a d_{f}^{q} g_{i}, \quad 0 \leq q \leq k-1,1 \leq i \leq m\right\}, k=1, \ldots, n
$$

with $a d_{f}^{0} g_{i}=g_{i}$ and $a d_{f}^{l} g_{i}=\left[f, a d_{f}^{l-1} g_{i}\right]$ for all $l \geq 1$.

Theorem 1.1 (i) A control system $\Sigma: \dot{x}=f(x)+g(x) u$ is locally state equivalent to a linear controllable system $\Lambda: \dot{w}=F w+G u$ if and only if

(S1) $\operatorname{dim} \operatorname{span}\left\{g(x), a d_{f} g(x), \ldots, a d_{f}^{n-1} g(x)\right\}=n$;

(S2) $\left[a d_{f}^{q} g, a d_{f}^{r} g\right]=0, \quad 0 \leq q<r \leq n$.

(ii) A control system $\Sigma: \dot{x}=f(x)+g(x) u$ is locally equivalent, via a feedback transformation $\Gamma=(\phi, \alpha, \beta)$ to a linear controllable system $\Lambda: \dot{w}=A w+b v$ if and only if (F1) $\operatorname{dim} \operatorname{span}\left\{g(x), a d_{f} g(x), \ldots, a d_{f}^{n-1} g(x)\right\}=n$;

(F2) $\mathcal{D}^{n-1}$ is involutive, that is, $\left[\mathcal{D}^{n-1}, \mathcal{D}^{n-1}\right] \subseteq \mathcal{D}^{n-1}$.

If the transformation $\Gamma=(\phi, \alpha, \beta)$ linearizes $\Sigma$, then $(P D E s)$ should hold with $\tilde{f}(\phi(x))=A \phi(x), \tilde{g}(\phi(x))=B$. Although the conditions $(S 1)$ and $(S 2)$ (resp. $(F 1)$ and $(F 2)$ ) provide a way of testing the state (resp. feedback) linearizability of a system, they offer little on how to find the state (resp. feedback) linearizing group $\Gamma$ except by solving ( $P D E s)$ which is, in general, not straightforward. Indeed, for the single-input case, the solvability of (PDEs) is equivalent of finding a function $h$ with $h(0)=0$ such that

$L_{g}(h)=0, L_{g} L_{f}(h)=0, \ldots, L_{g} L_{f}^{n-2}(h)=0, L_{g} L_{f}^{n-1}(h) \neq 0$,

where for any vector field $\nu$ and any function $h, L_{\nu}(h)=$ $\frac{\partial h}{\partial x} v(x)$ is the Lie derivative of $h$ along $\nu$. We propose here to give a complete solution to both problem 1 and 
problem 2 without solving the corresponding partial differential equations. We will provide an algorithm giving explicit solutions in each case. Recall that we have previously obtained explicit solutions for few subclasses of control-affine systems, namely strict feedforward forms, strict-feedforward nice and feedforward forms, for which linearizing coordinates were found without solving the corresponding PDEs (see [28], [29], [31]). Indeed, for those subclasses we exhibited algorithms that can be performed using a maximum of $\frac{n(n+1)}{2}$ steps each involving composition and integration of functions only (but not solving PDEs) followed by a sequence of $n+1$ derivations. What played a main role in finding those algorithms were the strict feedforward form structure, that is, the fact that each component of the system depended only on higher variables. In this paper we consider general control-affine systems for which we provide a state and a feedback linearizing algorithms that can be implemented each using a maximum of $n$ steps. Those algorithms are, in part, based on the explicit solving of the flow-box theorem [32] and differ completely from those outlined in [28], [31] (see also [18], [19]). Another approach was proposed in [24] based on successive integrations of differential 1-forms. It relies on successive rectification of vector fields via the characteristic method using quotient manifolds in order to reduce, at each step, the dimension of the system by one. The difference between our approach and the later is two fold: (a) explicit formulas are given in term of convergent series without solving any PDE or ODE; (b) the algorithm provides a sequence of control-affine systems without restriction on any manifold or performing a quotient on some direction. We will address here the single input case; the generalization to multiple-input control systems is in consideration and expected to appear somewhere. Let us mention that the linearization techniques have been very useful and are still of interest nowadays. If Problem 1 or Problem 2 is solvable with a controllable pair $(A, b)$, then the equilibrium of $\Sigma$ can be stabilized by the feedback law $u=-\beta(x)^{-1}\left(\alpha(x)+\sum_{j=1}^{n} k_{j} \varphi_{j}(x)\right)$, where the polynomial $p(\lambda)=\lambda^{n}+\sum_{j=1}^{n} k_{j} \lambda^{j-1}$ is Hurwitz. This can be used to improve the dynamical behavior of chaotic systems as it can be seen for the Lorenz control system in [26]. Feedback linearization techniques have also been applied to optimal control problems (e.g. minimizing time) and have regained some interest recently. In [7] the authors used pseudospectral method to solve optimal control problem of feedback linearizable dynamics subject to mixed state and control constraints. As mentioned by the authors, such problems frequently arise in astronautical applications where stringent performance requirements demand optimality over feedback linearizing controls. Mayer's problem has also been considered in [1] (see also [26]) and an optimal solution for globally feedback linearizable time-invariant systems, subject to path and actuator constraints, obtained. Recall that Mayer's problem consists of determining $u(t)$ and $x(t)$ with $t \in\left[t_{0}, t_{f}\right]$ that minimize a functional cost $J=\Phi\left(x\left(t_{f}\right), t_{f}\right)$ subject to the dynamics $\dot{x}=f(x)+g(x) u$ and inequality constraints $\tilde{s}(x, u) \leq 0, \tilde{c}(x) \leq 0$ when initial states are given and terminal states satisfy $\Psi\left(x\left(t_{0}\right), x\left(t_{f}\right)\right)=0$. In all these problems however, either the dynamics are assumed to be already linear or a linearizing coordinate is known through the natural outputs. Let mention that due to the difficulty of solving the partial differential equations in one part, and the fact many systems are not feedback linearizable, the exact feedback linearization has been extended in various ways. The notions of partial linearization, approximate linearization, pseudo-linearization, extended linearization, etc, have been introduced in the literature [3], [5], [6], [8], $[9],[15],[17],[21],[36]$ to off-set the difficulties associated with exact linearization. Partial linearization is thought when the system fails to satisfy the integrability conditions and relies on the idea of finding the largest subsystem that can be linearizable. Approximate linearization was first developed in [17] and later generalized in [15] using Taylor series expansions up to some degree. The changes of coordinates and feedback obtained in this case are polynomial that linearizes the system up to some degree, and their obtention relies on a step-by-step algorithm or by the use of outputs of the system defining a relative degree for the system. In many of the methods proposed, the integrability conditions are either weakened or they are applied to a specific class of systems (we refer the interested reader to [5] for a survey and the references therein). The paper is organized as following. In Section 1.3 we give some definitions and notations to be used throughout the paper. The first main result on state linearization is given in Section 2 where an algorithm is presented, and the feedback case considered in Section 4. Illustrative examples follow each section and are given in Section 3 and Section 5. A constructive solution of the flow box theorem as well as the convergence of the series is presented in Section 6 followed by a conclusion.

\subsection{Notations and Definitions}

For simplicity of exposition we first consider single-input control systems

$$
\Sigma: \dot{x}=f(x)+g(x) u, x \in \mathbb{R}^{n}, u \in \mathbb{R} .
$$

The case of multi-input systems is more involved and will be addressed somewhere else. Let $0 \leq k \leq n-1$ be an integer.

Definition 1.2 We say that $\Sigma$ is Brunovský $k$-linear if

$$
g(x)=b, a_{f} g(x)=A b, \ldots, a d_{f}^{n-k-1} g(x)=A^{n-k-1} b,
$$

where $(A, b)$ is the Brunovský canonical pair.

We will denote hereafter the coordinates in which the system is Brunovský $k$-linear by the bolded variables $\mathbf{x}_{\mathbf{k}}=$ 
$\left(\mathbf{x}_{\mathbf{k} 1}, \ldots, \mathbf{x}_{\mathbf{k} n}\right)^{\mathrm{T}}$ and the system by $\Sigma_{\mathbf{k}}^{\mathrm{Br}}$, where $k=\mathbf{k}$. It follows easily that a Brunovský $k$-linear system takes the form

$$
\Sigma_{\mathbf{k}}^{\mathrm{Br}}:\left\{\begin{aligned}
\dot{\mathbf{x}}_{\mathbf{k} j} & =F_{\mathbf{k} j}\left(\mathbf{x}_{\mathbf{k} 1}, \ldots, \mathbf{x}_{\mathbf{k} k+1}\right), \text { if } 1 \leq j \leq k \\
\dot{\mathbf{x}}_{\mathbf{k} k+1} & =F_{\mathbf{k} k+1}\left(\mathbf{x}_{\mathbf{k} 1}, \ldots, \mathbf{x}_{\mathbf{k} k+1}\right)+\mathbf{x}_{\mathbf{k} k+2} \\
& \ldots \\
\dot{\mathbf{x}}_{\mathbf{k} n-1} & =F_{\mathbf{k} n-1}\left(\mathbf{x}_{\mathbf{k} 1}, \ldots, \mathbf{x}_{\mathbf{k} k+1}\right)+\mathbf{x}_{\mathbf{k} n} \\
\dot{\mathbf{x}}_{\mathbf{k} n} & =F_{\mathbf{k} n}\left(\mathbf{x}_{\mathbf{k} 1}, \ldots, \mathbf{x}_{\mathbf{k} k+1}\right)+u
\end{aligned}\right.
$$

A more compact representation of $\Sigma_{\mathbf{k}}^{\mathrm{Br}}$ is obtained as

$$
\Sigma_{\mathbf{k}}^{\mathrm{Br}}: \dot{\mathbf{x}}_{\mathbf{k}}=F_{\mathbf{k}}\left(\mathbf{x}_{\mathbf{k} 1}, \ldots, \mathbf{x}_{\mathbf{k} k+1}\right)+A \hat{\mathbf{x}}_{\mathbf{k}}+b u, \mathbf{x}_{\mathbf{k}} \in \mathbb{R}^{n},
$$

where $\hat{\mathbf{x}}_{\mathbf{k}}=\left(0, \ldots, 0, \mathbf{x}_{\mathbf{k} k+2}, \mathbf{x}_{\mathbf{k} k+3}, \ldots, \mathbf{x}_{\mathbf{k} n}\right)^{\mathrm{T}}$ is a vector whose first $k+1$ components are zero. The Brunovský $k$-linear forms will play a crucial role in the state linearization algorithm. For the feedback linearization algorithm in Section 4 , the Brunovský $k$-linear forms are replaced by the feedback $k$-forms defined as following.

Definition 1.3 A control-affine system $\Sigma: \dot{x}=f(x)+$ $g(x) u$ is said to be in $(F B)_{\mathbf{k}}$-form, and we denote it $\Sigma_{\mathbf{k}}^{\mathrm{FB}}$, if in some coordinates $\mathbf{x}_{\mathbf{k}}=\left(\mathbf{x}_{\mathbf{k} 1}, \ldots, \mathbf{x}_{\mathbf{k} n}\right)^{\mathrm{T}}$, it takes the form

$\Sigma_{\mathbf{k}}^{\mathrm{FB}}:\left\{\begin{aligned} \dot{\mathbf{x}}_{\mathbf{k} j} & =F_{\mathbf{k} j}\left(\mathbf{x}_{\mathbf{k} 1}, \ldots, \mathbf{x}_{\mathbf{k} k+1}\right), \text { if } 1 \leq j \leq k \\ \dot{\mathbf{x}}_{\mathbf{k} k+1} & =F_{\mathbf{k} k+1}\left(\mathbf{x}_{\mathbf{k} 1}, \ldots, \mathbf{x}_{\mathbf{k} k+2}\right) \\ & \ldots \\ \dot{\mathbf{x}}_{\mathbf{k} n-1} & =F_{\mathbf{k} n-1}\left(\mathbf{x}_{\mathbf{k} 1}, \ldots, \mathbf{x}_{\mathbf{k} n}\right) \\ \dot{\mathbf{x}}_{\mathbf{k} n} & =F_{\mathbf{k} n}\left(\mathbf{x}_{\mathbf{k} 1}, \ldots, \mathbf{x}_{\mathbf{k} n}\right)+u,\end{aligned}\right.$

where $k=\mathbf{k}$. For simplicity we chose the coefficient of the control input $u$ to be 1 but this is not a restriction.

\section{Main Results: $\mathcal{S}$-Linearizability}

The first result is as follows and states that any $\mathcal{S}$ linearizable system can be transformed into a linear form via a sequence of explicit coordinates changes each giving rise to a Brunovský k-linear system.

Theorem 2.1 Consider a controllable system

$$
\Sigma: \dot{x}=f(x)+g(x) u, x \in \mathbb{R}^{n}, u \in \mathbb{R} .
$$

Assume it is $\mathcal{S}$-linearizable (denote $\Sigma \triangleq \Sigma_{\mathbf{n}}^{\mathrm{Br}}$ and $x \triangleq \mathbf{x}_{\mathbf{n}}$ ). There exists a sequence of explicit coordinates changes $\varphi_{\mathbf{n}}\left(\mathbf{x}_{\mathbf{n}}\right), \varphi_{\mathbf{n}-\mathbf{1}}\left(\mathbf{x}_{\mathbf{n}-\mathbf{1}}\right), \ldots, \varphi_{\mathbf{1}}\left(\mathbf{x}_{\mathbf{1}}\right)$ that gives rise to a sequence of Brunovský $\mathbf{k}$-linear systems $\Sigma_{\mathbf{n}}^{\mathrm{Br}}, \Sigma_{\mathbf{n}-\mathbf{1}}^{\mathrm{Br}}, \ldots, \Sigma_{\mathbf{0}}^{\mathrm{Br}}$ such that $\Sigma_{\mathbf{k}-\mathbf{1}}^{\mathrm{Br}}=\varphi_{\mathbf{k} *}\left(\Sigma_{\mathbf{k}}^{\mathrm{Br}}\right)$ for any $1 \leq \mathbf{k} \leq n$. The Brunovský $\mathbf{k}$-linear system $\Sigma_{\mathbf{k}}^{\mathrm{Br}}$ is mapped into the Brunovský $(\mathbf{k}-\mathbf{1})$-linear system $\Sigma_{\mathbf{k}-\mathbf{1}}^{\mathrm{Br}}$ if and only if

$$
\left(\mathcal{S} £_{k+1}\right) \triangleq \frac{\partial^{2} F_{\mathbf{k}}}{\partial \mathbf{x}_{\mathbf{k} k+1}^{2}}=0 .
$$

Moreover, in the coordinates $w \triangleq \varphi_{\mathbf{1}}\left(\mathbf{x}_{\mathbf{1}}\right)$ the system $\Sigma$ (actually $\Sigma_{\mathbf{0}}^{\mathrm{Br}}$ ) takes the simpler linear form

$$
\Lambda_{\lambda}: \dot{w}=A_{\lambda} w+b u \triangleq\left\{\begin{array}{rll}
\dot{w}_{1} & = & \lambda_{1} w_{1}+w_{2} \\
\dot{w}_{2} & = & \lambda_{2} w_{1}+w_{3} \\
& \ldots & \\
\dot{w}_{n-1} & =\lambda_{n-1} w_{1}+w_{n} \\
\dot{w}_{n} & =\lambda_{n} w_{1}+u,
\end{array}\right.
$$

where $\lambda_{1}, \ldots, \lambda_{n}$ are constant real numbers.

The condition (2.1) remains the main criteria for the linearizing algorithm; it is a simplified version of Theorem 1.1 (S2). It barely means that the nonlinear vector field $F_{\mathbf{k}}\left(\mathbf{x}_{\mathbf{k} 1}, \ldots, \mathbf{x}_{\mathbf{k} k+1}\right)$ should be affine with respect to the variable $\mathbf{x}_{\mathbf{k} k+1}$. At each step, we need to check if that condition is satisfied, then proceed if yes and stop otherwise. The proof of this theorem relies mainly on the flow-box theorem for which we gave recently explicit solution [32] (see below) and on Theorem 1.1 (S2).

Theorem 2.2 Let $\nu$ be a smooth vector field on $\mathbb{R}^{n}$, any integer $1 \leq k \leq n$ such that $\nu_{k}(0) \neq 0$ and $\sigma_{k}(x)=1 / \nu_{k}(x)$.

(i) Define $z=\varphi(x)$ by its components as following

$$
\begin{aligned}
\varphi_{j}(x) & =x_{j}+\sum_{s=1}^{\infty} \frac{(-1)^{s} x_{k}^{s}}{s !} L_{\sigma_{k} \nu}^{s-1}\left(\sigma_{k} \nu_{j}\right)(x) \\
\varphi_{k}(x) & =\sum_{s=1}^{\infty} \frac{(-1)^{s+1} x_{k}^{s}}{s !} L_{\sigma_{k} \nu}^{s-1}\left(\sigma_{k}\right)(x)
\end{aligned}
$$

for any $1 \leq j \leq n, j \neq k$. The diffeomorphism $z=\varphi(x)$ satisfies $\varphi_{*}(\nu)=\partial_{z_{k}}$.

(ii) The diffeomorphism $x=\psi(z)$ given by its components

$$
\begin{aligned}
& \psi_{j}(z)=z_{j}+\sum_{s=1}^{\infty} \frac{z_{k}^{s}}{s !}\left(\sum_{i=0}^{s-1}(-1)^{i} C_{s}^{i} \partial_{z_{k}}^{i} L_{\nu}^{s-i-1}\left(\nu_{j}\right)(z)\right) \\
& \psi_{k}(z)=\sum_{s=1}^{\infty} \frac{z_{k}^{s}}{s !}\left(\sum_{i=0}^{s-1}(-1)^{i} C_{s}^{i} \partial_{z_{k}}^{i} L_{\nu}^{s-i-1}\left(\nu_{k}\right)(z)\right)
\end{aligned}
$$

for any $1 \leq j \leq n, j \neq k$, is the inverse of $z=\varphi(x)$, that is, such that $\frac{\partial \psi(z)}{\partial z_{k}}=\nu(\psi(z))$.

Above, $\varphi_{*}(\nu)=\frac{\partial \varphi}{\partial x}\left(\varphi^{-1}(z)\right) \nu\left(\varphi^{-1}(z)\right)$ is the mapping of tangent space induced by the diffeomorphism $z=\varphi(x)$, and we have adopted the following notation

$$
\partial_{z_{k}}=\frac{\partial}{\partial z_{k}}, \partial_{z_{k}} \cdot h=\frac{\partial h}{\partial z_{k}}, \ldots, \partial_{z_{k}}^{i} \cdot h=\frac{\partial^{i} h}{\partial z_{k}^{i}}, i \geq 2 .
$$

The following remarks are of paramount importance here. R1. The expressions above are not series around the origin or in the variable $x_{k}$ as the coefficients $L_{\sigma_{k} \nu}^{s}\left(\sigma_{k} \nu_{j}\right)(x)$ are 
evaluated at $x=\left(x_{1}, \ldots, x_{n}\right)$ and might well depend on $x_{k}$.

R2. If the vector field $\nu$ is independent of some variable $x_{l}$ $(l \neq k)$, then the diffeomorphism $\varphi(x)$ is also independent of the variable $x_{l}$ (except a linear dependence).

R3. If any of the components of $\nu(x)$ is zero, say $\nu_{j}(x)=0$, then $\varphi_{j}(x)=x_{j}$.

A proof of the theorem and the convergence of the series will be given in Section 6. In Section 3 we illustrate with few examples, in particular Example 3.4 will justify the fact that the expressions (2.2)-(2.3) of Theorem 2.2 are not Taylor series at the origin. For further details we refer to $[32]$.

\subsection{Linearizing Coordinates}

In this section we define an algorithm that shows how to compute the linearizing coordinates for the system. The algorithm stands also as a proof of Theorem 2.1. Although the algorithm generates a sequence of new coordinates $\mathbf{x}_{\mathbf{n}}, \mathbf{x}_{\mathbf{n}-\mathbf{1}}, \ldots, \mathbf{x}_{\mathbf{1}}$ as stated in Theorem 2.1, at each step, say Step $n-\mathbf{k}$, we will reset the coordinates of the system as $x$, i.e., set $x=\mathbf{x}_{\mathbf{k}}$ and take the coordinates of its transform as $z$, i.e., put $z=\mathbf{x}_{\mathbf{k}-\mathbf{1}}$. Moreover, the corresponding system $\Sigma_{\mathbf{k}}^{\mathrm{Br}}$ will be renamed as $\Sigma: \dot{x}=f(x)+g(x) u$ and its transform $\Sigma_{\mathbf{k}-\mathbf{1}}^{\mathrm{Br}}$ by $\tilde{\Sigma}: \dot{z}=\tilde{f}(z)+\tilde{g}(z) u$.

A. $(\mathcal{S} £)$-Algorithm. Consider a linearly controllable system

$$
\Sigma: \dot{x}=f(x)+g(x) u, x \in \mathbb{R}^{n}, u \in \mathbb{R} .
$$

Without loss of generality take $g(0)=b=(0, \ldots, 0,1)^{\mathrm{T}}$. This algorithm consists of $n-1$ steps.

Step 0. Set $\Sigma \triangleq \Sigma_{\mathbf{n}}^{\mathrm{Br}}$ and $x \triangleq \mathbf{x}_{\mathbf{n}}=\left(\mathbf{x}_{\mathbf{n} 1}, \ldots, \mathbf{x}_{\mathbf{n} n}\right)^{\mathrm{T}}$. Apply Theorem 2.2 with $\nu=g(x)$ to construct a change of coordinates $z=\varphi(x)$ given by (2.2), such that $\varphi_{*}(g)(z)=\partial z_{n}$. Such change of coordinates transforms $\Sigma$ into

$$
\tilde{\Sigma}: \dot{z}=\tilde{f}(z)+\tilde{g}(z) u=\left(\varphi_{*} f\right)(z)+\left(\varphi_{*} g\right)(z) u, z \in \mathbb{R}^{n},
$$

where $\tilde{g}=b$. Denote $\mathbf{x}_{\mathbf{n}-\mathbf{1}} \triangleq z$ and $\varphi_{\mathbf{n}} \triangleq \varphi$. It follows that the change of coordinates $\mathbf{x}_{\mathbf{n}-\mathbf{1}}=\varphi_{\mathbf{n}}\left(\mathbf{x}_{\mathbf{n}}\right)$ transforms $\Sigma_{\mathbf{n}}^{\mathrm{Br}}$ into

$$
\Sigma_{\mathbf{n}-\mathbf{1}}^{\mathrm{Br}}: \dot{\mathbf{x}}_{\mathbf{n}-\mathbf{1}}=F_{\mathbf{n}-\mathbf{1}}\left(\mathbf{x}_{\mathbf{n}-\mathbf{1}}\right)+A \hat{\mathbf{x}}_{\mathbf{n}-\mathbf{1}}+b u, \mathbf{x}_{\mathbf{n}-\mathbf{1}} \in \mathbb{R}^{n}
$$

where $\hat{\mathbf{x}}_{\mathbf{n}-\mathbf{1}} \equiv 0$ and $F_{\mathbf{n}-\mathbf{1}}\left(\mathbf{x}_{\mathbf{n}-\mathbf{1}}\right)=\tilde{f}\left(\mathbf{x}_{\mathbf{n}-\mathbf{1}}\right)=\varphi_{\mathbf{n} *}(f)$. Step 1. Reset the variable $x \triangleq \mathbf{x}_{\mathbf{n}-\mathbf{1}}$ and $\Sigma \triangleq \Sigma_{\mathbf{n}-\mathbf{1}}^{\mathrm{Br}}$ : $f(x)+g(x) u$ with $g(x)=b$ and $f(x)=F_{\mathbf{n}-\mathbf{1}}\left(x_{1}, \ldots, x_{n}\right)$. For $\Sigma$ to be $\mathcal{S}$-linearizable, Theorem 1.1 (S2) should be satisfied, which is equivalent to

$$
\left[a d_{f}^{q} g, a d_{f}^{r} g\right]=0, \quad 0 \leq q, r \leq n-1
$$

Taking $q=0$ and $r=1$ we get in particular $\left[g, a d_{f} g\right]=0$ or equivalently (because $g=\partial x_{n}$ )

$$
\left(\mathcal{S} £_{n}\right) \triangleq \frac{\partial^{2} f}{\partial x_{n}^{2}}=0 .
$$

It follows that $f$ should be affine with respect to the variable $x_{n}$. If this condition fails then the system is not $\mathcal{S}$ linearizable and the algorithm stops. Otherwise, the vector field $f$ decomposes uniquely as

$$
f\left(x_{1}, \ldots, x_{n}\right)=F_{\mathbf{n}-1}\left(x_{1}, \ldots, x_{n-1}\right)+x_{n} \nu\left(x_{1}, \ldots, x_{n-1}\right) .
$$

Because $g, a d_{f} g$ are linearly independent, then $\nu(0) \neq 0$. Apply Theorem 2.2 to define a change of coordinates $z=\varphi(x)$ such that $\varphi_{*}(\nu)=\partial_{z_{n-1}}$. Denote $z \triangleq \mathbf{x}_{n-2}$ and $\varphi \triangleq \varphi_{\mathbf{n}-\mathbf{1}}$. The diffeomorphism $\mathbf{x}_{\mathbf{n}-\mathbf{2}}=\varphi_{\mathbf{n}-\mathbf{1}}\left(\mathbf{x}_{\mathbf{n}-\mathbf{1}}\right)$ transforms $\Sigma_{\mathbf{n}-\mathbf{1}}^{\mathrm{Br}}$ into

$$
\Sigma_{\mathbf{n}-\mathbf{2}}^{\mathrm{Br}}: \dot{\mathbf{x}}_{\mathbf{n}-\mathbf{2}}=F_{\mathbf{n}-\mathbf{2}}\left(\mathbf{x}_{\mathbf{n}-\mathbf{2}}\right)+A \hat{\mathbf{x}}_{\mathbf{n}-\mathbf{2}}+b u, \mathbf{x}_{\mathbf{n}-\mathbf{2}} \in \mathbb{R}^{n},
$$

where $\hat{\mathbf{x}}_{\mathbf{n}-\mathbf{2}}=\left(0, \ldots, 0, \mathbf{x}_{\mathbf{n}-\mathbf{2} n}\right)^{\mathrm{T}}$ and $F_{\mathbf{n}-\mathbf{2}}\left(\mathbf{x}_{\mathbf{n}-\mathbf{2}}\right)=$ $\varphi_{\mathbf{n}-2 *}\left(F_{\mathbf{n}-1}\right)$ is function of the variables $\mathbf{x}_{\mathbf{n}-21}, \ldots, \mathbf{x}_{\mathbf{n}-2 n-1}$.

Step $n-\mathbf{k}$. Assume that $\Sigma_{\mathbf{n}}^{\mathrm{Br}}$ has been taken, via a composition $\mathbf{x}_{\mathbf{k}}=\varphi_{\mathbf{k}+\mathbf{1}} \circ \cdots \circ \varphi_{\mathbf{n}}(x)$ of diffeomorphisms, into

$$
\Sigma_{\mathbf{k}}^{B r}: \dot{\mathbf{x}}_{\mathbf{k}}=F_{\mathbf{k}}\left(\mathbf{x}_{\mathbf{k} 1}, \ldots, \mathbf{x}_{\mathbf{k} k+1}\right)+A \hat{\mathbf{x}}_{\mathbf{k}}+b u, \mathbf{x}_{\mathbf{k}} \in \mathbb{R}^{n},
$$

where $\hat{\mathbf{x}}_{\mathbf{k}}=\left(0, \ldots, 0, \mathbf{x}_{\mathbf{k} k+2}, \mathbf{x}_{\mathbf{k} k+3}, \ldots, \mathbf{x}_{\mathbf{k} n}\right)^{\mathrm{T}}$, and the last $n-k$ components of the vector field $F_{\mathbf{k}}\left(\mathbf{x}_{\mathbf{k}}\right)$ are zero. Once again reset the variable $x \triangleq \mathbf{x}_{\mathbf{k}}$ and denote $\Sigma_{\mathbf{k}}^{\mathrm{Br}}$ simply by $\Sigma: \dot{x}=f(x)+g(x) u$ with $g(x)=b$ and

$$
f(x)=F_{\mathbf{k}}\left(x_{1}, \ldots, x_{k+1}\right)+A \hat{x}_{k}
$$

where $\hat{x}_{k}=\left(0, \ldots, 0, x_{k+2}, x_{k+3}, \ldots, x_{n}\right)^{\mathrm{T}}$. Notice that in these coordinates

$g=\partial_{x_{n}}, a d_{f} g=-\partial_{x_{n-1}}, \ldots, a d_{f}^{n-k-1} g=(-1)^{n-k-1} \partial_{x_{k+1}}$

which implies that $a d_{f}^{n-k} g=(-1)^{n-k} \frac{\partial F_{\mathbf{k}}}{\partial x_{k+1}}$. For $r=n-$ $k-1$ and $q=r+1$, the condition $\left[a d_{f}^{q} g, a d_{f}^{r} g\right]=0$ of Theorem 1.1 (S1) is equivalent to

$$
\left(\mathcal{S} £_{k+1}\right) \triangleq \frac{\partial^{2} f}{\partial x_{k+1}^{2}}=\frac{\partial^{2} F_{\mathbf{k}}}{\partial x_{k+1}^{2}}=0 .
$$

If the condition fails to be satisfied, then the system is not state linearizable and the algorithm stops. If satisfied this means that $F_{\mathbf{k}}$ is affine with respect to the variable $x_{k+1}$ and decomposes as

$$
F_{\mathbf{k}}\left(x_{k}\right)=f_{k}\left(x_{1}, \ldots, x_{k}\right)+x_{k+1} \nu\left(x_{1}, \ldots, x_{k}\right),
$$

where $\nu$ is a nonsingular vector field in $\mathbb{R}^{n}$ that depends exclusively on the variables $x_{1}, \ldots, x_{k}$. By Theorem 2.2 we can construct a change of coordinates $z=\varphi(x)$ such that $\varphi_{*}(\nu)(z)=\partial_{z_{k}}$. Moreover the components of $\varphi$ are such that

$$
\varphi_{j}(x)=x_{j}+\phi_{j}\left(x_{1}, \ldots, x_{k}\right), 1 \leq j \leq n .
$$

This change of coordinates transforms $\Sigma$ into

$$
\tilde{\Sigma}: \dot{z}=\tilde{f}(z)+\tilde{g}(z) u=\left(\varphi_{*} f\right)(z)+\left(\varphi_{*} g\right)(z) u
$$


where $\tilde{g}(z)=\left(\varphi_{*} g\right)(z)=(0, \ldots, 0,1)^{\mathrm{T}}$ and

$\tilde{f}(z)=\left(\varphi_{*} F_{\mathbf{k}}\right)(z)+\left[z_{k+1}-\phi_{k+1}\left(\varphi^{-1}(z)\right)\right]\left(\varphi_{*} \nu\right)(z)+\varphi_{*}(A \hat{x})(z)$

Because the $k$ first components of $A \hat{x}$ are zero, then (2.5) implies $\varphi_{*}(A \hat{x})(z)=\left(0, \ldots, 0, z_{k+2}, \ldots, z_{n}, 0\right)^{\mathrm{T}}$. We then deduce that $\tilde{f}(z)=F_{\mathbf{k}-\mathbf{1}}(z)+A \tilde{z}$, where $F_{\mathbf{k}-\mathbf{1}}(z)=$ $\left(\varphi_{*} F_{\mathbf{k}}\right)(z)-\varphi_{k+1}\left(\varphi^{-1}(z)\right) \partial_{z_{k}}$ depends exclusively on the variables $z_{1}, \ldots, z_{k}$ and

$$
\begin{aligned}
A \hat{z} & =z_{k+1} \partial_{z_{k}}+\left(0, \ldots, 0, z_{k+2}, z_{k+3}, \ldots, z_{n}, 0\right)^{\mathrm{T}} \\
& =\left(0, \ldots, 0, z_{k+1}, z_{k+2}, \ldots, z_{n}, 0\right)^{\mathrm{T}}
\end{aligned}
$$

is such that the $k$ first components are zero. Notice that when $k=0$, the expression above reduces simply to

$$
F_{\mathbf{0}}(z)=z_{1} \lambda, \quad \text { where } \lambda=\left(\lambda_{1}, \ldots, \lambda_{n}\right)^{\mathrm{T}} .
$$

This ends the general step and shows that a sequence of explicit coordinates changes $\varphi_{\mathbf{n}}\left(\mathbf{x}_{\mathbf{n}}\right), \ldots, \varphi_{\mathbf{1}}\left(\mathbf{x}_{\mathbf{1}}\right)$ can be constructed whose composition $z=\varphi_{1} \circ \cdots \circ \varphi_{\mathbf{n}}\left(\mathbf{x}_{\mathbf{n}}\right)$ takes the original system $\Sigma$ into the linear form $\Lambda_{\lambda}$.

B. Summary of Algorithm. Start with a system

$$
\Sigma: \dot{x}=f(x)+g(x) u, x \in \mathbb{R}^{n}, u \in \mathbb{R} .
$$

Step 0. Normalize the vector field $g \longmapsto g=$ $(0, \ldots, 0,1)^{\mathrm{T}}$. Apply a linear change of coordinates to transform the linearization such that $\frac{\partial f}{\partial x}(0)=A_{\lambda}$.

Step $n-k$. If the condition

$$
\left(\mathcal{S} £_{k+1}\right) \triangleq \frac{\partial^{2} f}{\partial x_{k+1}^{2}}=0
$$

fails, the algorithm stops: The system is not $\mathcal{S}$-linearizable. If $\left(\mathcal{S} £_{k+1}\right)$ holds, then decompose the vector field $f$ as

$$
f\left(x_{1}, \ldots, x_{k+1}\right)=F\left(x_{1}, \ldots, x_{k}\right)+x_{k+1} \nu\left(x_{1}, \ldots, x_{k}\right) .
$$

Apply Theorem 2.2 to construct a change of coordinates $z=\varphi(x) \in \mathbb{R}^{n}$ that rectifies the nonsingular vector field

$$
\nu(x)=\nu_{1}(x) \partial_{x_{1}}+\cdots+\nu_{n}(x) \partial_{x_{n}}
$$

that is, such that $\varphi_{*}(\nu)(z)=\partial_{z_{k}}$. Find the transform $\varphi_{*} \Sigma$ of the system in precedent step. For $k=n-1, n-2, \ldots, 2$ repeat Step $n-k$. End if system is linear or algorithm fails.

\section{State Linearization: Examples}

In what follows we illustrate with few examples.

Example 3.1 Consider a single-input control system

$\Sigma: \dot{x}=f(x)+g(x) u \triangleq\left\{\begin{array}{l}\dot{x}_{1}=x_{2}-2 x_{2} x_{3}+x_{3}^{2}+4 x_{2} x_{3} u \\ \dot{x}_{2}=x_{3}-2 x_{3} u \\ \dot{x}_{3}=u\end{array}\right.$ with $f(x)=\left(x_{2}-2 x_{2} x_{3}+x_{3}^{2}, x_{3}, 0\right)^{\mathrm{T}}$ and $g(x)=$ $\left(4 x_{2} x_{3},-2 x_{3}, 1\right)^{\mathrm{T}}$. First rectify the vector field $\nu(x) \triangleq g(x)$ $z)$. by applying Theorem 2.2 with $n=3$ and $\sigma_{3}(x)=1$. Since

$$
L_{\nu}\left(\nu_{1}\right)=-8 x_{3}^{2}+4 x_{2}, L_{\nu}^{2}\left(\nu_{1}\right)=-24 x_{3}, L_{\nu}^{3}\left(\nu_{1}\right)=-24,
$$

we have $L_{\nu}^{s-1}\left(\nu_{1}\right)=0$ for all $s \geq 5$ and hence

$$
\begin{aligned}
z_{1}=\varphi_{1}(x) & =x_{1}+\sum_{s=1}^{\infty}(-1)^{s} \frac{x_{3}^{s}}{s !}\left(L_{\nu}^{s-1} \nu_{1}\right)(x) \\
& =x_{1}-4 x_{2} x_{3}^{2}-4 x_{3}^{4}+2 x_{2} x_{3}^{2}+4 x_{3}^{4}-x_{3}^{4} \\
& =x_{1}-2 x_{2} x_{3}^{2}-x_{3}^{4} .
\end{aligned}
$$

Likewise, $L_{\nu}\left(\nu_{2}\right)=-2$ and $L_{\nu}^{s-1}\left(\nu_{2}\right)=0, s \geq 3$, yielding

$$
\begin{aligned}
z_{2}=\varphi_{2}(x) & =x_{2}+\sum_{s=1}^{\infty}(-1)^{s} \frac{x_{3}^{s}}{s !}\left(L_{\nu}^{s-1} \nu_{2}\right)(x) \\
& =x_{2}-x_{3}\left(-2 x_{3}\right)+(1 / 2 !) x_{3}^{2}(-2)=x_{2}+x_{3}^{2} .
\end{aligned}
$$

We apply the change of coordinates

$$
z_{1}=x_{1}-2 x_{2} x_{3}^{2}-x_{3}^{4}, z_{2}=x_{2}+x_{3}^{2}, z_{3}=x_{3}
$$

to transform the original system into

$$
\tilde{\Sigma}: \dot{z}=\tilde{f}(z)+\tilde{g}(z) u \triangleq\left\{\begin{aligned}
\dot{z}_{1} & =z_{2}-2 z_{2} z_{3} \\
\dot{z}_{2} & =z_{3} \\
\dot{z}_{3} & =u
\end{aligned}\right.
$$

where $\tilde{g}(z)=(0,0,1)^{\mathrm{T}}$ and $\tilde{f}(z)=\left(z_{2}-2 z_{2} z_{3}, z_{3}, 0\right)^{\mathrm{T}}$. The vector field $\tilde{f}(z)=\left(z_{2}-2 z_{2} z_{3}, z_{3}, 0\right)^{\mathrm{T}}$ decomposes

$$
\tilde{f}(z)=\left(z_{2}, 0,0\right)^{\mathrm{T}}+z_{3}\left(-2 z_{2}, 1,0\right)^{\mathrm{T}} .
$$

The next step is to rectify $\nu(x)=\left(-2 z_{2}, 1,0\right)^{\mathrm{T}}$. Theorem 2.2 with $k=2$ and $\sigma_{2}(z)=1$ yields

$$
\begin{aligned}
w_{1} & =z_{1}+\sum_{s=1}^{\infty}(-1)^{s} \frac{z_{2}^{s}}{s !} L_{\nu}^{s-1}\left(\nu_{1}\right)(z) \\
& =z_{1}-z_{2}\left(-2 z_{2}\right)+(1 / 2 !) z_{2}^{2}(-2)=z_{1}+z_{2}^{2} \\
w_{2} & =z_{2} \\
w_{3} & =z_{3} .
\end{aligned}
$$

The system is then transformed, under these change of coordinates, to the linear Brunovský form $\Lambda_{B r}$. The linearizing coordinates for the original system are thus obtained as a composition of the two-step coordinate changes

$$
\begin{aligned}
& w_{1}=x_{1}-2 x_{2} x_{3}^{2}-x_{3}^{4}+\left(x_{2}+x_{3}^{2}\right)^{2}=x_{1}+x_{2}^{2} \\
& w_{2}=x_{2}+x_{3}^{2} \\
& w_{3}=x_{3} .
\end{aligned}
$$

Of course, these linearizing coordinates could have been obtained directly or by other methods. The emphasis here is on the applicability of the method to any linearizable system. 
Example 3.2 We consider the following example

$\Sigma: \dot{x}=f(x)+g(x) u \triangleq\left\{\begin{array}{l}\dot{x}_{1}=x_{2}+\left((1 / 2) x_{2}-(1 / 12) x_{3} x_{4}\right) u \\ \dot{x}_{2}=x_{3}+(1 / 2) x_{3} u \\ \dot{x}_{3}=x_{4}+x_{4} u \\ \dot{x}_{4}=u .\end{array}\right.$

Because of the strict feedforward structure, we showed in [28] (using a 4-step algorithm) that the change of coordinates

$z=\varphi(x) \triangleq\left\{\begin{aligned} z_{1} & =x_{1}-(1 / 24)\left(12 x_{2} x_{4}-4 x_{3} x_{4}^{2}+x_{4}^{4}\right) \\ z_{2} & =x_{2}-(1 / 2)\left(x_{3} x_{4}-(1 / 3) x_{4}^{3}\right) \\ z_{3} & =x_{3}-(1 / 2) x_{4}^{2} \\ z_{4} & =x_{4}\end{aligned}\right.$

linearizes the system. We can recover such coordinates directly by applying the algorithm given in the proof. Denote by $f(x)=\left(x_{2}, x_{3}, x_{4}, 0\right)^{\mathrm{T}}$ and

$$
\nu(x) \triangleq g(x)=\left((1 / 2) x_{2}-(1 / 12) x_{3} x_{4},(1 / 2) x_{3}, x_{4}, 1\right)^{\mathrm{T}} .
$$

The first step consists of rectifying the control vector field via Theorem 2.2. Since $\nu_{3}=1$, hence $\sigma_{3}=1$ we have

$L_{\nu}\left(\nu_{1}\right)=(1 / 2)\left(x_{3} / 2\right)-(1 / 12)\left(x_{4}^{2}+x_{3}\right)=(1 / 6) x_{3}-(1 / 12) x_{4}^{2}$, and $L_{\nu}^{2}\left(\nu_{1}\right)=\frac{1}{6} x_{4}-\frac{1}{6} x_{4}=0$, i.e., $L_{\nu}^{s}\left(\nu_{1}\right)=0, s \geq 2$. Thus

$$
\begin{aligned}
\varphi_{1}(x) & =x_{1}-x_{4} \nu_{1}(x)+(1 / 2) x_{4}^{2} L_{\nu}\left(\nu_{1}\right) \\
& =x_{1}-(1 / 2) x_{2} x_{4}+(1 / 6) x_{3} x_{4}^{2}-(1 / 24) x_{4}^{3} .
\end{aligned}
$$

Also $L_{\nu}\left(\nu_{2}\right)=\frac{1}{2} x_{4}, L_{\nu}^{2}\left(\nu_{2}\right)=\frac{1}{2}$ and $L_{\nu}^{s}\left(\nu_{2}\right)=0, s \geq 3$ implies

$$
\begin{aligned}
\varphi_{2}(x) & =x_{2}-x_{4} \nu_{2}(x)+(1 / 2) x_{4}^{2} L_{\nu}\left(\nu_{2}\right)-(1 / 6) x_{4}^{3} L_{\nu}^{2}\left(\nu_{2}\right) \\
& =x_{2}-(1 / 2) x_{3} x_{4}+(1 / 4) x_{4}^{3}-(1 / 12) x_{4}^{3} \\
& =x_{2}-(1 / 2) x_{3} x_{4}+(1 / 6) x_{4}^{3} .
\end{aligned}
$$

Similarly $L_{\nu}\left(\nu_{3}\right)=1$ and $L_{\nu}^{s-1}\left(\nu_{3}\right)=0, \forall s \geq 3$. Hence

$$
\begin{aligned}
\varphi_{3}(x) & =x_{3}-x_{4} \nu_{3}(x)+(1 / 2) x_{4}^{2} L_{\nu}\left(\nu_{2}\right) \\
& =x_{3}-x_{4}^{2}+(1 / 2) x_{4}^{2}=x_{3}-(1 / 2) x_{4}^{2} .
\end{aligned}
$$

Because $\nu_{4}(x)=1$, we get $\varphi_{4}(x)=x_{4}$ and the change of coordinates (3.1) rectifies the control vector field $g$ and linearizes the system. Notice that the algorithm described in [28] allowed only to find (3.1) by computing one component at a time (holding other components identity), starting from $\varphi_{3}$ then $\varphi_{2}$ and finally $\varphi_{1}$ and updating the system after each step. A composition of different coordinates changes gave (3.1). However, Theorem 2.2 allows to compute those components independently to each other.

Example 3.3 Consider $\nu(x)=x_{3} \partial_{x_{1}}+\left(x_{2}+x_{3}\right) \partial_{x_{2}}+\partial_{x_{3}}$ in $\mathbb{R}^{3}$. Here $L_{\nu}\left(\nu_{1}\right)=1$ and $L_{\nu}^{s-1}\left(\nu_{1}\right)=0$ for $s \geq 3$ and $L_{\nu}^{s-1}\left(\nu_{2}\right)=x_{2}+x_{3}+1$ for all $s \geq 2$. It follows that

$$
\begin{aligned}
\varphi_{1}(x) & =x_{1}+\sum_{s=1}^{\infty} \frac{(-1)^{s} x_{3}^{s}}{s !} L_{\nu}^{s-1}\left(\nu_{1}\right)(x) \\
& =x_{1}-x_{3} \nu_{1}(x)+(1 / 2 !) x_{3}^{2} L_{\nu}\left(\nu_{1}\right)(x) \\
& =x_{1}-(1 / 2) x_{3}^{2}
\end{aligned}
$$

and

$$
\begin{aligned}
\varphi_{2}(x) & =x_{2}+\sum_{s=1}^{\infty} \frac{(-1)^{s} x_{3}^{s}}{s !} L_{\nu}^{s-1}\left(\nu_{2}\right)(x) \\
& =x_{2}-x_{3} \nu_{2}(x)+\sum_{s=2}^{\infty} \frac{(-1)^{s} x_{3}^{s}}{s !}\left(x_{2}+x_{3}+1\right) \\
& =\left(x_{2}+x_{3}+1\right) e^{-x_{3}}-1 .
\end{aligned}
$$

To find the inverse first notice that $\partial_{z_{3}}^{i} \cdot L_{\nu}^{s-i-1}\left(\nu_{1}\right)(z)=0$ if $(i, s) \neq(0,1)$, which yields

$$
\begin{aligned}
\psi_{1}(z) & =z_{1}+\sum_{s=1}^{\infty} \frac{z_{3}^{s}}{s !}\left(\sum_{i=0}^{s-1}(-1)^{i} C_{s}^{i} \partial_{z_{n}}^{i} L_{\nu}^{s-i-1}\left(\nu_{1}\right)(z)\right) \\
& =z_{1}+(1 / 2 !) z_{3}^{2} \nu_{1}(z)=z_{1}+(1 / 2) z_{3}^{2} .
\end{aligned}
$$

From $\partial_{z_{3}}^{i} \cdot L_{\nu}^{s-i-1}\left(\nu_{2}\right)(z)=0$ for all $i \geq 2$, we deduce

$$
\begin{aligned}
& \sum_{i=0}^{s-1}(-1)^{i} C_{s}^{i} \partial_{z_{3}}^{i} L_{\nu}^{s-i-1}\left(\nu_{2}\right)(z) \\
& =L_{\nu}^{s-1}\left(\nu_{2}\right)(z)-s \partial_{z_{3}} L_{\nu}^{s-2}\left(\nu_{2}\right)(z)=z_{2}+z_{3}+1-s .
\end{aligned}
$$

By Theorem 2.2 (ii) we get the $2^{\text {nd }}$ component of $\psi(z)$ as

$$
\begin{aligned}
\psi_{2}(z) & =z_{2}+\sum_{s=1}^{\infty} \frac{z_{3}^{s}}{s !}\left(\sum_{i=0}^{s-1}(-1)^{i} C_{s}^{i} \partial_{z_{3}}^{i} L_{\nu}^{s-i-1}\left(\nu_{2}\right)(z)\right) \\
& =z_{2}+\sum_{s=1}^{\infty} \frac{z_{3}^{s}}{s !}\left(z_{2}+z_{3}+1\right)-\sum_{s=1}^{\infty} \frac{z_{3}^{s}}{s !} s \\
& =\left(z_{2}+1\right) e^{z_{3}}-z_{3}-1 .
\end{aligned}
$$

It is straightforward to verify that the inverse is

$$
x=\varphi^{-1}(z) \triangleq\left\{\begin{array}{l}
x_{1}=\psi_{1}(z)=z_{1}+(1 / 2) z_{3}^{2} \\
x_{2}=\psi_{2}(z)=\left(z_{2}+1\right) e^{z_{3}}-z_{3}-1 \\
x_{3}=\psi_{3}(z)=z_{3} .
\end{array}\right.
$$

Example 3.4 Consider the non singular vector field $\nu(x)=\lambda\left(x_{3}\right) \partial_{x_{1}}+\partial_{x_{3}}, \quad x \in \mathbb{R}^{3}$, where $\lambda$ is a flat function, that is, $\lambda$ and all its derivatives are zero at $x_{3}=0$. A well-known example is the function defined by $\lambda(0)=0$, and $\lambda\left(x_{3}\right)=\exp \left(-1 / x_{3}^{2}\right)$ if $x_{3} \neq 0$. It is straightforward to check that $L_{\nu}^{s-1}\left(\nu_{1}\right)(x)=\lambda^{(s-1)}\left(x_{3}\right)$ for all $s \geq 1$, where $\lambda^{(k)}\left(x_{3}\right)$ is the $k$ th derivative of $\lambda$. Should (2.2) have been a series around 0 or at $x_{k}=0$ the straightening diffeomorphism would have been identity:

$z=\varphi(x) \triangleq\left\{\begin{array}{l}\varphi_{1}(x)=x_{1}+\sum_{s=1}^{\infty} \frac{(-1)^{s} x_{3}^{s}}{s !} L_{\nu}^{s-1}\left(\nu_{1}\right)(0)=x_{1} \\ \varphi_{2}(x)=x_{2}+\sum_{s=1}^{\infty} \frac{(-1)^{s} x_{3}^{s}}{s !} L_{\nu}^{s-1}\left(\nu_{2}\right)(0)=x_{2} \\ \varphi_{3}(x)=\sum_{s=1}^{\infty} \frac{(-1)^{s-1} x_{3}^{s}}{s !} L_{\nu}^{s-1}(1)(0)=x_{3}\end{array}\right.$ which is impossible. However we can verify easily that $\varphi_{1}(x)=x_{1}-\int_{0}^{x_{3}} \lambda(u) \mathrm{d} u$ which coincides with

$$
\varphi_{1}(x)=x_{1}+\sum_{s=1}^{\infty} \frac{(-1)^{s} x_{3}^{s}}{s !} \lambda^{(s-1)}\left(x_{3}\right) .
$$


Indeed, $\int_{0}^{x_{3}} \lambda(u) \mathrm{d} u=-\sum_{s=1}^{\infty} \frac{(-1)^{s} x_{3}^{s}}{s !} \lambda^{(s-1)}\left(x_{3}\right)$ because the two functions coincide when $x_{3}=0$ and it is enough to verify that their derivatives are also equal. The derivative of the right hand side gives after simplification

$$
-\sum_{s=1}^{\infty} \frac{(-1)^{s} x_{3}^{s-1}}{(s-1) !} \lambda^{(s-1)}\left(x_{3}\right)-\sum_{s=1}^{\infty} \frac{(-1)^{s} x_{3}^{s}}{s !} \lambda^{(s)}\left(x_{3}\right)=\lambda\left(x_{3}\right) .
$$

Now to find the inverse of the normalizing coordinates, let us apply Theorem 2.2 (ii) with $n=3$ and $k=3$. First we have $L_{\nu}^{s} \nu=\lambda^{(s)}\left(x_{3}\right) \partial_{x_{1}}$ for all $s \geq 1$. We thus have

$$
\begin{aligned}
\psi(z) & =z+\sum_{s=1}^{\infty} \frac{z_{3}^{s}}{s !}\left(\sum_{i=0}^{s-1}(-1)^{i} C_{s}^{i} \partial_{z_{3}}^{i}\left(L_{\nu}^{s-i-1} \nu\right)(z)\right) \\
& =z+\sum_{s=1}^{\infty} \frac{z_{3}^{s}}{s !}\left(\sum_{i=0}^{s-1}(-1)^{i} C_{s}^{i}\right) \lambda^{(s-1)}\left(z_{3}\right) \partial_{z_{1}} \\
& =\left(\begin{array}{c}
z_{1}-\sum_{s=1}^{\infty} \frac{(-1)^{s} z_{3}^{s}}{s !} \lambda^{(s-1)}\left(z_{3}\right) \\
z_{2} \\
z_{3}
\end{array}\right)
\end{aligned}
$$

It clearly follows that $\psi(z)=\left(z_{1}+\int_{0}^{z_{3}} \lambda(s) \mathrm{d} s, z_{2}, z_{3}\right)^{\mathrm{T}}$ which was predictable directly by inverting $z=\varphi(x)$. $\quad$

\section{Main Results: $\mathcal{F}$-Linearizable Systems}

Below we give our main result, that is, an algorithm allowing to construct explicitly feedback linearizing coordinates. We first recall the following well-known result.

Theorem 4.1 A control system $\Sigma: \dot{x}=f(x)+g(x) u$ is locally $\mathcal{F}$-equivalent to a linear controllable system if and only if it is $\mathcal{S}$-equivalent to a feedback form

$$
\left\{\begin{aligned}
\dot{z}_{1} & =\hat{f}_{1}\left(z_{1}, z_{2}\right) \\
\dot{z}_{2} & =\hat{f}_{2}\left(z_{1}, z_{2}, z_{3}\right) \\
& \ldots \\
\dot{z}_{n-1} & =\hat{f}_{n-1}\left(z_{1}, \ldots, z_{n}\right) \\
\dot{z}_{n} & =\hat{f}_{n}\left(z_{1}, \ldots, z_{n}\right)+\hat{g}_{n}\left(z_{1}, \ldots, z_{n}\right) u
\end{aligned}\right.
$$

The proof of Theorem 4.1 is straightforward and can be found in the literature (e.g. [11], [12], [13], [25]). Let $\hat{f}=$ $\left(\hat{f}_{1}, \ldots, \hat{f}_{n}\right), \hat{g}=\left(0, \ldots, 0, \hat{g}_{n}\right)$ and $\hat{h}(z)=z_{1}$. It follows that the feedback transformation $\Gamma \triangleq(\hat{\varphi}, \hat{\alpha}, \hat{\beta})$ defined by $w=\hat{\varphi}(z), u=\hat{\alpha}(z)+\hat{\beta}(z) v$, where

$$
\begin{gathered}
\hat{\varphi}_{1}(z)=\hat{h}(z), \hat{\varphi}_{2}(z)=L_{\hat{f}}(\hat{h}), \ldots, \hat{\varphi}_{n}(z)=L_{\hat{f}}^{n-1}(\hat{h}) \\
\hat{\alpha}(z)=-\frac{L_{\hat{f}}^{n}(\hat{h})}{L_{\hat{g}} L_{\hat{f}}^{n-1}(\hat{h})} \text { and } \hat{\beta}(z)=-\frac{1}{L_{\hat{g}} L_{\hat{f}}^{n-1}(\hat{h})}
\end{gathered}
$$

brings $(F B)$ into the Brunovský canonical form $\Lambda_{B r}$. Consider $\Sigma: \dot{x}=f(x)+g(x) u$ and recall Definition 1.3 that $\Sigma$ is in $(F B)_{\mathbf{k}}$-form, if in some coordinates $\mathbf{x}_{\mathbf{k}}=$ $\left(\mathbf{x}_{\mathbf{k} 1}, \ldots, \mathbf{x}_{\mathbf{k} n}\right)$, it takes the form

$\Sigma_{\mathbf{k}}^{\mathrm{FB}}:\left\{\begin{aligned} \dot{\mathbf{x}}_{\mathbf{k} j} & =F_{\mathbf{k} j}\left(\mathbf{x}_{\mathbf{k} 1}, \ldots, \mathbf{x}_{\mathbf{k} k+1}\right), \text { if } 1 \leq j \leq k \\ \dot{\mathbf{x}}_{\mathbf{k} k+1} & =F_{\mathbf{k} k+1}\left(\mathbf{x}_{\mathbf{k} 1}, \ldots, \mathbf{x}_{\mathbf{k} k+2}\right) \\ & \ldots \\ \dot{\mathbf{x}}_{\mathbf{k} n-1} & =F_{\mathbf{k} n-1}\left(\mathbf{x}_{\mathbf{k} 1}, \ldots, \mathbf{x}_{\mathbf{k} n}\right) \\ \dot{\mathbf{x}}_{\mathbf{k} n} & =F_{\mathbf{k} n}\left(\mathbf{x}_{\mathbf{k} 1}, \ldots, \mathbf{x}_{\mathbf{k} n}\right)+u,\end{aligned}\right.$

where $k=\mathbf{k}$.

Theorem 4.2 Consider a linearly controllable system

$$
\Sigma: \dot{x}=f(x)+g(x) u, x \in \mathbb{R}^{n}, u \in \mathbb{R} .
$$

Assume it is $\mathcal{F}$-linearizable (let $\Sigma \triangleq \Sigma_{\mathbf{n}}^{F B}$ and $x \triangleq \mathbf{x}_{\mathbf{n}}$ ). There exists a sequence of explicit coordinates changes $\varphi_{\mathbf{n}}\left(\mathbf{x}_{\mathbf{n}}\right), \varphi_{\mathbf{n}-\mathbf{1}}\left(\mathbf{x}_{\mathbf{n}-\mathbf{1}}\right), \ldots, \varphi_{\mathbf{2}}\left(\mathbf{x}_{\mathbf{2}}\right)$ that gives rise to a sequence of $(F B)_{\mathbf{k}}$-forms $\Sigma_{\mathbf{n}-\mathbf{1}}^{\mathrm{FB}}, \Sigma_{\mathbf{n}-\mathbf{2}}^{\mathrm{FB}}, \ldots, \Sigma_{\mathbf{1}}^{\mathrm{FB}}$ such that for any $2 \leq \mathbf{k} \leq n$ we get $\Sigma_{\mathbf{k}-\mathbf{1}}^{\mathrm{FB}}=\left(\varphi_{\mathbf{k}}\right)_{*} \Sigma_{\mathbf{k}}^{\mathrm{FB}}$. Moreover, in the coordinates $z \triangleq \varphi_{\mathbf{2}}\left(\mathbf{x}_{\mathbf{2}}\right)$ the system $\Sigma$ (actually $\Sigma_{\mathbf{1}}^{\mathrm{FB}}$ ) takes the feedback form $(F B)$.

A direct consequence of this result is the following corollary.

Corollary 4.3 Consider a linearly controllable system $\Sigma$ and assume it is $\mathcal{F}$-linearizable. Then $\Sigma$ is linearizable by the feedback transformation $w=\hat{\varphi} \circ \varphi(x), u=\hat{\alpha}(\varphi(x))+$ $\hat{\beta}(\varphi(x)) v$, where $z=\varphi(x)$ is the diffeomorphism taking $\Sigma$ into the feedback form $(F B)$, and $\Gamma=(\hat{\varphi}, \hat{\alpha}, \hat{\beta})$ the transformation taking $(F B)$ into to the Brunovsky form $\Lambda_{B r}$.

The proof of Theorem 4.2 follows from the algorithm below.

A. $(\mathcal{F} £)$-Linearizing Algorithm. Consider the system $\Sigma: \dot{x}=f(x)+g(x) u, x \in \mathbb{R}^{n}, u \in \mathbb{R}$ and assume it is $\mathcal{F}$-linearizable. Applying a linear feedback $z=T x, u=$ $K x+L v$, if necessary, we assume that $\frac{\partial f}{\partial x}(0)=A$ and $g(0)=b$, where $(A, b)$ is the Brunovský canonical pair. The algorithm below consists of a maximum of $n-1$ steps. Step 1. Set $\Sigma \triangleq \Sigma_{\mathbf{n}}^{\mathrm{FB}}$ and $x \triangleq \mathbf{x}_{\mathbf{n}}=\left(\mathbf{x}_{\mathbf{n} 1}, \ldots, \mathbf{x}_{\mathbf{n} n}\right)^{\mathrm{T}}$. Apply Theorem II.2 ([33]) with $\nu=g(x)$ to construct a change of coordinates $z=\varphi(x)$ such that $\varphi_{*}(g)(z)=\partial_{z_{n}}$. If we denote $\mathbf{x}_{\mathbf{n}-\mathbf{1}} \triangleq z$ and $\varphi_{\mathbf{n}} \triangleq \varphi$, it thus follows that the change of coordinates $\mathbf{x}_{\mathbf{n}-\mathbf{1}}=\varphi_{\mathbf{n}}\left(\mathbf{x}_{\mathbf{n}}\right)$ takes $\Sigma_{\mathbf{n}}^{\mathrm{FB}}$ into

$$
\Sigma_{\mathbf{n}-\mathbf{1}}^{\mathrm{FB}}:\left\{\begin{array}{rll}
\dot{\mathbf{x}}_{\mathbf{n}-\mathbf{1 1}} & =F_{\mathbf{n}-\mathbf{1 1}}\left(\mathbf{x}_{\mathbf{n}-\mathbf{1} 1}, \ldots, \mathbf{x}_{\mathbf{n}-\mathbf{1} n}\right) \\
\dot{\mathbf{x}}_{\mathbf{n}-\mathbf{1 2}} & =F_{\mathbf{n}-\mathbf{1 2}}\left(\mathbf{x}_{\mathbf{n}-\mathbf{1 1}}, \ldots, \mathbf{x}_{\mathbf{n}-\mathbf{1} n}\right) \\
& \ldots \\
\dot{\mathbf{x}}_{\mathbf{n}-\mathbf{1} n-1} & =F_{\mathbf{n}-\mathbf{1} n-1}\left(\mathbf{x}_{\mathbf{n}-\mathbf{1 1}}, \ldots, \mathbf{x}_{\mathbf{n}-\mathbf{1} n}\right) \\
\dot{\mathbf{x}}_{\mathbf{n}-\mathbf{1} n} & =F_{\mathbf{n}-\mathbf{1} n}\left(\mathbf{x}_{\mathbf{n}-\mathbf{1 1}}, \ldots, \mathbf{x}_{\mathbf{n}-\mathbf{1} n}\right)+u .
\end{array}\right.
$$

Remark that this first step is independent of whether $\Sigma$ is $\mathcal{F}$-linearizable or not. It depends only on the fact that the vector field $g$ is nonsingular, and hence, can be rectified. 
Step $n-k$. Assume that a sequence of explicit coordinates changes $\varphi_{\mathbf{n}}, \ldots, \varphi_{\mathbf{k}+\mathbf{1}}$ were found whose composition $\mathbf{x}_{\mathbf{k}}=$ $\varphi_{\mathbf{k}+\mathbf{1}} \circ \cdots \circ \varphi_{\mathbf{n}}\left(\mathbf{x}_{\mathbf{n}}\right)$ takes $\Sigma_{\mathbf{n}}^{\mathrm{FB}}$ into the $(F B)_{\mathbf{k}}$-form

$$
\Sigma_{\mathbf{k}}^{\mathrm{FB}}: \dot{\mathbf{x}}_{\mathbf{k}}=F_{\mathbf{k}}\left(\mathbf{x}_{\mathbf{k}}\right)+b u, \mathbf{x}_{\mathbf{k}} \in \mathbb{R}^{n},
$$

where (recall that $k=\mathbf{k}$ )

$$
F_{\mathbf{k} j}\left(\mathbf{x}_{\mathbf{k}}\right)= \begin{cases}F_{\mathbf{k} j}\left(\mathbf{x}_{\mathbf{k} 1}, \ldots, \mathbf{x}_{\mathbf{k} k+1}\right), & 1 \leq j \leq k \\ F_{\mathbf{k} j}\left(\mathbf{x}_{\mathbf{k} 1}, \ldots, \mathbf{x}_{\mathbf{k} j+1}\right), & k+1 \leq j \leq n-1 \\ F_{\mathbf{k} j}\left(\mathbf{x}_{\mathbf{k} 1}, \ldots, \mathbf{x}_{\mathbf{k} n}\right), & j=n .\end{cases}
$$

Once again reset the variable $x \triangleq \mathbf{x}_{\mathbf{k}}$ and denote $\Sigma_{\mathbf{k}}^{\mathrm{FB}}$ simply by $\Sigma: \dot{x}=f(x)+g(x) u$ with $g(x)=b$ and

$$
f_{j}(x)= \begin{cases}f_{j}\left(x_{1}, \ldots, x_{k+1}\right), & 1 \leq j \leq k \\ f_{j}\left(x_{1}, \ldots, x_{j+1}\right), & k+1 \leq j \leq n,\end{cases}
$$

where the last component $f_{n}$ depends only on $x_{1}, \ldots, x_{n}$. We showed in Section 6 (6.1) that there exist smooth functions $\Theta(x)=\Theta\left(x_{1}, \ldots, x_{k+1}\right), F_{j}(x)=F_{j}\left(x_{1}, \ldots, x_{k}\right)$ and $\nu_{j}(x)=\nu_{j}\left(x_{1}, \ldots, x_{k}\right)$ for $1 \leq j \leq k$ such that $f_{j}\left(x_{1}, \ldots, x_{k+1}\right)=F_{j}(x)+\nu_{j}(x) \Theta(x) 1 \leq j \leq k$ with $\Theta(0) \neq 0$. Moreover, $\nu_{k}(0) \neq 0$ because $\frac{\partial f_{k}}{\partial x_{k+1}}(0) \neq 0$. Define the nonsingular vector field $\nu(x)=\nu_{1}(x) \partial_{x_{1}}+\cdots+$ $\nu_{k}(x) \partial_{x_{k}} \in \mathbb{R}^{k}$ and apply Theorem II.2 ([33]) to construct a change of coordinates $z=\varphi\left(x_{1}, \ldots, x_{k}\right) \in \mathbb{R}^{k}$ such that $\varphi_{*}(\nu)(z)=\partial_{z_{k}}$. Extend such change of coordinates in $\mathbb{R}^{n}$ (still called $\varphi$ ) by

$$
z=\varphi(x)=\left(\varphi_{1}(x), \ldots, \varphi_{k}(x), x_{k+1}, \ldots, x_{n}\right)^{\mathrm{T}} .
$$

The inverse $x=\psi(z)=\varphi^{-1}(z)$ is also obtained by Theorem II.2 ([33]). Clearly, the inverse is of the form

$$
x=\psi(z)=\left(\psi_{1}(z), \ldots, \psi_{k}(z), z_{k+1}, \ldots, z_{n}\right)^{\mathrm{T}} .
$$

The change of coordinates transforms the system $\Sigma$ into

$$
\tilde{\Sigma}: \dot{z}=\tilde{f}(z)+\tilde{g}(z) u=\varphi_{*} f(z)+\varphi_{*} g(z) u,
$$

where $\varphi_{*} g(z)=(0, \ldots, 0,1)^{\mathrm{T}}$ and

$$
\begin{aligned}
\tilde{f}(z)= & \varphi_{*} f(z)=\sum_{j=1}^{k} \varphi_{*}\left(f_{j}\left(x_{1}, \ldots, x_{k+1}\right) \partial_{x_{j}}\right) \\
& +\sum_{j=k+1}^{n} \varphi_{*}\left(f_{j}\left(x_{1}, \ldots, x_{j+1}\right) \partial_{x_{j}}\right) .
\end{aligned}
$$

It is easy to see that the second term is equivalent to

$$
\sum_{j=k+1}^{n} \varphi_{*}\left(f_{j}\left(x_{1}, \ldots, x_{j+1}\right) \partial_{x_{j}}\right)=\sum_{j=k+1}^{n} f_{j}(\psi(z)) \partial_{z_{j}} .
$$

The first term rewrites

$$
\begin{aligned}
\sum_{j=1}^{k} \varphi_{*}\left(f_{j}(x) \partial_{x_{j}}\right)= & \sum_{j=1}^{k} \varphi_{*}\left(F_{j}\left(x_{1}, \ldots, x_{k}\right) \partial_{x_{j}}\right) \\
& +\sum_{j=1}^{k} \varphi_{*}\left(\Theta(x) \nu_{j}\left(x_{1}, \ldots, x_{k}\right) \partial_{x_{j}}\right) \\
= & \sum_{j=1}^{k} \tilde{F}_{j}\left(z_{1}, \ldots, z_{k}\right) \partial_{z_{j}}+\Theta(\psi(z)) \partial_{z_{k}}
\end{aligned}
$$

We deduce from (4.2) that the first $k-1$ components depend only on the variables $z_{1}, \ldots, z_{k}$ and the $k$ th component depends on $z_{1}, \ldots, z_{k+1}$. In the other hand (4.1) shows that the $j$ th component $(j=k+1, \ldots, n)$ depends on the variables $z_{1}, \ldots, z_{j+1}$. We thus conclude that

$$
\tilde{f}_{j}(z)= \begin{cases}\tilde{f}_{j}\left(z_{1}, \ldots, z_{k}\right), & 1 \leq j \leq k-1 \\ \tilde{f}_{j}\left(z_{1}, \ldots, z_{j+1}\right), & k \leq j \leq n\end{cases}
$$

where the last component $\tilde{f}_{n}$ depends only on $z_{1}, \ldots, z_{n}$. Denote $\mathbf{x}_{\mathbf{k}-\mathbf{1}} \triangleq z$ and $\varphi_{\mathbf{k}} \triangleq \varphi$. Thus the change of coordinates $\mathbf{x}_{\mathbf{k}-\mathbf{1}}=\varphi_{\mathbf{k}}\left(\mathbf{x}_{\mathbf{k}}\right)$ brings the system $\Sigma_{\mathbf{k}}^{\mathrm{FB}}$ into

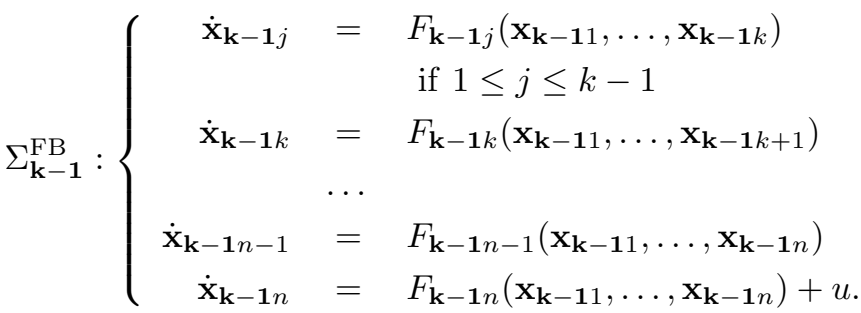

This completes the induction an the algortihm; consequently, we can construct a sequence of explicit coordinates changes $\varphi_{\mathbf{n}}\left(\mathbf{x}_{\mathbf{n}}\right), \varphi_{\mathbf{n}-\mathbf{1}}\left(\mathbf{x}_{\mathbf{n}-\mathbf{1}}\right), \ldots, \varphi_{\mathbf{2}}\left(\mathbf{x}_{\mathbf{2}}\right)$ whose composition $z=\varphi_{\mathbf{2}} \circ \cdots \circ \varphi_{\mathbf{n}}\left(\mathbf{x}_{\mathbf{n}}\right)$ takes the original system $\Sigma$ into the $(F B)$ form.

B. Summary of Algorithm. Start with a system $\Sigma: \dot{x}=$ $f(x)+g(x) u, x \in \mathbb{R}^{n}, u \in \mathbb{R}$.

Step 0. Normalize the vector field $g \longmapsto g=(0, \ldots, 0,1)^{\mathrm{T}}$ and apply a linear feedback to put the linearization in Brunovský form (not necessary but very recommended). Step $n-k$. If the condition

$$
\left(\mathcal{F} £_{k+1}\right) \triangleq \frac{\partial^{2} f_{j}}{\partial x_{k+1}^{2}}=\gamma_{n-k}(x) \frac{\partial f_{j}}{\partial x_{k+1}}, 1 \leq j \leq k
$$

fails $\left(\gamma_{n-k}(x)\right.$ not the same for first $k$ components) then system is not feedback linearizable and algorithm stops. If $\left(\mathcal{F} £_{k+1}\right)$ is satisfied, then decompose the first $k$ components $f_{1}, \ldots, f_{k}$ as following (see (6.1))

$$
f_{j}\left(x_{1}, \ldots, x_{k+1}\right)=F_{j}(x)+\nu_{j}(x) \Theta(x) 1 \leq j \leq k .
$$

Apply Theorem II.2 ([33]) to construct a change of coordinates $z=\varphi(x) \in \mathbb{R}^{n}$ to rectify the nonsingular vector field $\nu(x)=\nu_{1}(x) \partial_{x_{1}}+\cdots+\nu_{k}(x) \partial_{x_{k}}+0 \cdot \partial_{x_{k+1}}+\cdots+0 \cdot \partial_{x_{n}}$, that is, such that $\varphi_{*}(\nu)(z)=\partial_{z_{k}}$. Compute $\varphi_{*} \Sigma$ the transform of precedent system. Repeat Step $n-k$ for $k=n-1, \ldots, 2$. End if system is in (FB) form or algorithm fails.

\section{Feedback Linearization: Examples}

Example 5.1 Consider a single-input control system

$$
\Sigma: \dot{x}=f(x)+g(x) u \triangleq\left\{\begin{aligned}
\dot{x}_{1} & =x_{2}\left(1+x_{3}\right) \\
\dot{x}_{2} & =x_{3}\left(1+x_{1}\right)-x_{2} u \\
\dot{x}_{3} & =x_{1}+\left(1+x_{3}\right) u
\end{aligned}\right.
$$


with $f(x)=\left(x_{2}\left(1+x_{3}\right), x_{3}\left(1+x_{1}\right), x_{1}\right)^{\mathrm{T}}$ and $g(x)=$ $\left(0,-x_{2}, 1+x_{3}\right)^{\mathrm{T}}$. We first rectify the vector field $g(x)$. Put $\nu(x)=g(x)$ and apply Theorem II.2 ([33]) with $n=3$ and $\sigma_{3}(x)=\left(1+x_{3}\right)^{-1}$, thus $\sigma_{3} \nu=-x_{2}\left(1+x_{3}\right)^{-1} \partial_{x_{2}}+\partial_{x_{3}}$. Since $\nu_{1}=0$ and $\nu_{2}(x)=-x_{2}$, we have $\varphi_{1}(x)=x_{1}$ in one side, and

$L_{\sigma_{3} \nu}\left(\sigma_{3} \nu_{2}\right)=2 x_{2}\left(1+x_{3}\right)^{-2}, \quad L_{\sigma_{3} \nu}^{2}\left(\sigma_{3} \nu_{2}\right)=-6 x_{2}\left(1+x_{3}\right)^{-3}$

in the other, and recurrently

$$
L_{\sigma_{3} \nu}^{s-1}\left(\sigma_{3} \nu_{2}\right)=(-1)^{s} s ! x_{2}\left(1+x_{3}\right)^{-s} .
$$

It follows that

$z_{2}=\varphi_{2}(x)=x_{3}+\sum_{s=1}^{\infty} \frac{(-1)^{s} x_{3}^{s}}{s !} L_{\sigma_{3} \nu}^{s-1}\left(\sigma_{3} \nu_{2}\right)(x)=x_{2}\left(1+x_{3}\right)$.

To calculate $\varphi_{3}(x)$, notice that $L_{\sigma_{3} \nu}\left(\sigma_{3}\right)=-\left(1+x_{3}\right)^{-2}$ and $L_{\sigma_{3} \nu}^{2}\left(\sigma_{3}\right)=2\left(1+x_{3}\right)^{-3}$. Thus a simple recurrence shows that $L_{\sigma_{3} \nu}^{s-1} \sigma_{3}=(-1)^{s-1}(s-1) !\left(1+x_{3}\right)^{-s}$, for $s \geq 1$ which implies

$$
\begin{aligned}
z_{3}=\varphi_{3}(x) & =\sum_{s=1}^{\infty} \frac{(-1)^{s+1} x_{3}^{s}}{s !} L_{\sigma_{3} \nu}^{s-1}\left(\sigma_{3}\right)(x) \\
& =\sum_{s=1}^{\infty} \frac{1}{s}\left(\frac{x_{3}}{1+x_{3}}\right)^{s} \\
& ==\sum_{s=1}^{\infty} \int\left(\frac{x_{3}}{1+x_{3}}\right)^{s-1}\left(\frac{x_{3}}{1+x_{3}}\right)^{\prime} \mathrm{d} x_{3} \\
& =\int \frac{1}{1+x_{3}} \mathrm{~d} x_{3}=\ln \left(1+x_{3}\right) .
\end{aligned}
$$

We apply the change of coordinates $z_{1}=x_{1}, z_{2}=x_{2}(1+$ $\left.x_{3}\right), z_{3}=\ln \left(1+x_{3}\right)$ to transform the original system into

$\dot{z}=\hat{f}(z)+\hat{g}(z) u \triangleq\left\{\begin{array}{l}\dot{z}_{1}=z_{2} \\ \dot{z}_{2}=\left(1+z_{1}\right) e^{z_{3}}\left(e^{z_{3}}-1\right)+z_{1} z_{2} e^{-z_{3}} \\ \dot{z}_{3}=z_{1} e^{-z_{3}}+u .\end{array}\right.$

The system is in $(F B)$-form and can be put into the linear

Brunovský form $\Lambda_{B r}: \dot{w}_{1}=w_{2}, \dot{w}_{2}=w_{3}, \dot{w}_{3}=v$ via

$$
\left\{\begin{aligned}
w_{1} & =\hat{h}(z)=z_{1} \\
w_{2} & =L_{\hat{f}} \hat{h}(z)=z_{2} \\
w_{3} & =L_{\hat{f}}^{2} \hat{h}(z)=\left(1+z_{1}\right) e^{z_{3}}\left(e^{z_{3}}-1\right)+z_{1} z_{2} e^{-z_{3}} \\
v & =L_{\hat{f}}^{3} \hat{h}(z)+L_{\hat{g}} L_{\hat{f}}^{2} \hat{h}(z) u
\end{aligned}\right.
$$

The composition of the two-step changes of coordinates gives linearizing coordinates

$$
\left\{\begin{array}{l}
w_{1}=x_{1} \\
w_{2}=x_{2}\left(1+x_{3}\right) \\
w_{3}=x_{3}\left(1+x_{1}\right)\left(1+x_{3}\right)+x_{1} x_{2}
\end{array}\right.
$$

and feedback for the original system

$$
\begin{aligned}
v= & x_{2}\left(1+x_{3}\right)\left(x_{2}+x_{3}+x_{3}^{2}\right)+x_{1}\left(1+x_{1}\right)\left(1+3 x_{3}\right) \\
& +\left[\left(1+x_{1}\right)\left(1+x_{3}\right)\left(1+2 x_{3}\right)-x_{1} x_{2}\right] u
\end{aligned}
$$

Such linearizing coordinates and feedback could have been obtained by other methods. We want to point out that the method is applicable to all feedback linearizable systems.

Example 5.2 Consider a single-input control system

$$
\Sigma: \dot{x}=f(x)+g(x) u \triangleq\left\{\begin{array}{l}
\dot{x}_{1}=x_{2}-x_{4}^{2} \\
\dot{x}_{2}=x_{4}+2 x_{1}^{2} x_{4}+2 x_{4} u \\
\dot{x}_{3}=x_{1}^{2} \\
\dot{x}_{4}=x_{1}+x_{4}^{2}+u
\end{array}\right.
$$

with $f(x)=\left(x_{2}-x_{4}^{2}, x_{4}+2 x_{1}^{2} x_{4}, x_{1}^{2}, x_{1}+x_{4}^{2}\right)^{\mathrm{T}}$ and $g(x)=\left(0,2 x_{4}, 0,1\right)^{\mathrm{T}}$. This system is not feedback linearizable as it can be checked that $\left[g, a d_{f} g\right] \notin \operatorname{span}\left\{g, a d_{f} g\right\}$. We want to show that the algorithm provides such information without having to compute the involutivity of the distributions. We first start by rectifying the control vector field $g$. Identify $\nu=g(x)$ with $\sigma_{4}=1$. We calculate the component

$$
\begin{aligned}
\varphi_{2}(x) & =x_{2}+\sum_{s=1}^{\infty} \frac{(-1)^{s} x_{4}^{s}}{s !} L_{\nu}^{s-1}\left(\nu_{2}\right)(x) \\
& =x_{2}+\sum_{s=1}^{\infty} \frac{(-1)^{s} x_{4}^{s}}{s !} L_{\nu}^{s-1}\left(2 x_{4}\right)(x)=x_{2}-x_{4}^{2} .
\end{aligned}
$$

Since $\nu_{1}, \nu_{3}, \nu_{4}$ are constants, then $\varphi_{1}(x)=x_{1}, \varphi_{3}(x)=x_{3}$, and $\varphi_{4}(x)=x_{4}$. The change of coordinates $z_{1}=x_{1}, z_{2}=$ $x_{2}-x_{4}^{2}, z_{3}=x_{3}, z_{4}=x_{4}$ takes the system into

$\tilde{\Sigma}: \dot{z}=\tilde{f}(z)+\tilde{g}(z) u \triangleq\left\{\begin{aligned} \dot{z}_{1} & =z_{2} \\ \dot{z}_{2} & =z_{4}-2 z_{1} z_{4}+2 z_{1}^{2} z_{4}-2 z_{4}^{3} \\ \dot{z}_{3} & =z_{1}^{2} \\ \dot{z}_{4} & =z_{1}+z_{4}^{2}+u\end{aligned}\right.$

where $\tilde{g}=(0,0,0,1)^{\mathrm{T}}$ and

$$
\tilde{f}(z)=\left(z_{2}, z_{4}-2 z_{1} z_{4}+2 z_{1}^{2} z_{4}-2 z_{4}^{3}, z_{1}^{2}, z_{1}+z_{4}^{2}\right)^{\mathrm{T}} .
$$

Clearly,

$$
\frac{\partial \tilde{f}}{\partial z_{4}}=\left(0,1-2 z_{1}+2 z_{1}^{2}-6 z_{4}^{2}, 0,2 z_{4}\right)^{\mathrm{T}}, \frac{\partial^{2} \tilde{f}}{\partial z_{4}^{2}}=\left(0,-12 z_{4}, 0,2\right)^{\mathrm{T}}
$$

from which we deduce that $\frac{\partial^{2} \tilde{f}_{j}}{\partial z_{4}^{2}}=\gamma_{1} \frac{\partial \tilde{f}_{j}}{\partial z_{4}}, 1 \leq j \leq 3$ fails. The algorithm ends: the system is not $\mathcal{F}$-linearizable.

Example 5.3 Consider the single-input control system $[12]$

$$
\Sigma: \dot{x}=f(x)+g(x) u \triangleq\left\{\begin{array}{l}
\dot{x}_{1}=e^{x_{2}} u \\
\dot{x}_{2}=x_{1}+x_{2}^{2}+e^{x_{2}} u \\
\dot{x}_{3}=x_{1}-x_{2}
\end{array}\right.
$$

with $f(x)=\left(0, x_{1}+x_{2}^{2}, x_{1}-x_{2}\right)^{\mathrm{T}}$ and $g(x)=\left(e^{x_{2}}, e^{x_{2}}, 0\right)^{\mathrm{T}}$. We first rectify the vector field $g(x)$. Denote $\nu(x)=g(x)$ and apply Theorem II.2 ([33]) with $n=3$ and $\sigma_{2}(x)=$ 
$e^{-x_{2}}$, hence $\sigma_{2} \nu=\partial_{x_{1}}+\partial_{x_{2}}$. Since $\nu_{3}=0$, then $\varphi_{3}(x)=$ $x_{3}$. Because $L_{\sigma_{2} \nu}^{s-1}\left(\sigma_{2} \nu_{1}\right)=0$ for all $s \geq 2$, we obtain

$$
\begin{aligned}
z_{1}=\varphi_{1}(x) & =x_{1}+\sum_{s=1}^{\infty} \frac{(-1)^{s} x_{2}^{s}}{s !} L_{\sigma_{2} \nu}^{s-1}\left(\sigma_{2} \nu_{1}\right)(x) \\
& =x_{1}-x_{2}\left(\sigma_{2} \nu_{1}\right)(x)=x_{1}-x_{2}
\end{aligned}
$$

To compute $\varphi_{2}$ notice that $L_{\sigma_{2} \nu}^{s-1}\left(\sigma_{2}\right)=(-1)^{s-1} e^{-x_{2}}$ for all $s \geq 2$. It thus follows that

$$
\begin{aligned}
z_{2}=\varphi_{2}(x) & =\sum_{s=1}^{\infty} \frac{(-1)^{s+1} x_{2}^{s}}{s !} L_{\sigma_{2} \nu}^{s-1}\left(\sigma_{2}\right)(x) \\
& =\sum_{s=1}^{\infty} \frac{x_{2}^{s}}{s !} e^{-x_{2}}=1-e^{-x_{2}} .
\end{aligned}
$$

The change of coordinates

$$
z=\varphi(x)=\left(x_{1}-x_{2}, 1-e^{-x_{2}}, x_{3}\right)^{\mathrm{T}}
$$

whose inverse $x=\psi(z)=\left(z_{1}-\ln \left(1-z_{2}\right),-\ln \left(1-z_{2}\right), z_{3}\right)^{\mathrm{T}}$ can be obtained directly or by applying Theorem II.2 (ii) (see [33]), takes the original system into

$$
\left\{\begin{array}{l}
\dot{z}_{1}=-z_{1}+\ln \left(1-z_{2}^{2}\right)-\left(\ln \left(1-z_{2}\right)\right)^{2} \\
\dot{z}_{2}=\left(1-z_{2}\right)\left[z_{1}-\ln \left(1-z_{2}^{2}\right)+\left(\ln \left(1-z_{2}\right)\right)^{2}\right]+u \\
\dot{z}_{3}=z_{1}
\end{array}\right.
$$

A permutation of the variables $\tilde{z}_{1}=z_{3}, \tilde{z}_{2}=z_{1}, \tilde{z}_{3}=z_{2}$ yields a system in feedback form

$(F B)\left\{\begin{array}{l}\dot{\tilde{z}}_{1}=\tilde{z}_{2} \\ \dot{\tilde{z}}_{2}=-\tilde{z}_{2}+\ln \left(1-\tilde{z}_{3}^{2}\right)-\left(\ln \left(1-\tilde{z}_{3}\right)\right)^{2} \\ \dot{\tilde{z}}_{3}=\left(1-\tilde{z}_{3}\right)\left[\tilde{z}_{2}-\ln \left(1-\tilde{z}_{3}^{2}\right)+\left(\ln \left(1-\tilde{z}_{3}\right)\right)^{2}\right]+u\end{array}\right.$

that can be linearized by

$$
\begin{aligned}
w_{1} & =\tilde{z}_{1} \\
w_{1} & =\tilde{z}_{2} \\
w_{3} & =-\tilde{z}_{2}+\ln \left(1-\tilde{z}_{3}^{2}\right)-\left(\ln \left(1-\tilde{z}_{3}\right)\right)^{2} \\
v & =\dot{w}_{3} .
\end{aligned}
$$

We thus deduce that the change of coordinates

$$
\begin{aligned}
w_{1} & =x_{3} \\
w_{2} & =x_{1}-x_{2} \\
w_{3} & =-x_{1}-x_{2}^{2} \\
v & =-2 x_{2}\left(x_{1}+x_{2}^{2}\right)-\left(1+2 x_{2}\right) e^{x_{2}} u
\end{aligned}
$$

brings $\Sigma$ into Brunovský $\Lambda_{B r}: \dot{w}_{1}=w_{2}, \dot{w}_{2}=w_{3}, \dot{w}_{3}=v$. Notice that such change of coordinates was given in [12]. However, the system was coupled with the given output $y=h(x)=x_{3}$ which made finding them straightforward.

\section{Appendix: Proofs of Results}

Below we establish an equivalence between the involutivity conditions of Theorem 1.1 and a sequence of easily computable conditions $\left(\mathcal{F} £_{n}\right), \ldots,\left(\mathcal{F} £_{1}\right)$ each stating the fact that the second derivative of $f$ with respect to some variable is proportional to its first derivative with respect to the same variable. This constitutes the core of the algorithm.

Simple Involutivity Conditions. Consider the system $\Sigma$ : $\dot{x}=f(x)+g(x) u$ and assume without loss of generality that $g(x)=(0, \ldots, 0,1)^{\mathrm{T}}$ and

$$
f_{j}(x)= \begin{cases}f_{j}\left(x_{1}, \ldots, x_{k+1}\right) & 1 \leq j \leq k \\ f_{j}\left(x_{1}, \ldots, x_{j+1}\right) & k+1 \leq j \leq n\end{cases}
$$

where $1 \leq k \leq n-1$ and $f_{n}$ depends only on $x_{1}, \ldots, x_{n}$. Claim: If the following distributions

$$
\mathcal{D}^{j}(x)=\operatorname{span}\left\{g(x), a d_{f} g(x) \ldots, a d_{f}^{j-1} g(x)\right\}, 1 \leq j \leq n
$$

are involutive, then there is a function $\gamma_{n-k}$ such that

$$
\left(\mathcal{F} £_{k+1}\right) \triangleq \frac{\partial^{2} f_{j}}{\partial x_{k+1}^{2}}=\gamma_{n-k}(x) \frac{\partial f_{j}}{\partial x_{k+1}}, 1 \leq j \leq k .
$$

Moreover, functions $\Theta(x)=\Theta\left(x_{1}, \ldots, x_{k+1}\right)$ and $F_{j}(x)=$ $F_{j}\left(x_{1}, \ldots, x_{k}\right)$ and $\nu_{j}(x)=\nu_{j}\left(x_{1}, \ldots, x_{k}\right)$ exist such that

$$
f_{j}\left(x_{1}, \ldots, x_{k+1}\right)=F_{j}(x)+\nu_{j}(x) \Theta(x) 1 \leq j \leq k
$$

with $\Theta(x)$ depending exclusively on $\gamma_{n-k}(x)$.

Proof: Remark that the vector field $f$ can be written as

$f(x)=\sum_{j=1}^{k} f_{j}\left(x_{1}, \ldots, x_{k+1}\right) \partial_{x_{j}}+\sum_{j=k+1}^{n} f_{j}\left(x_{1}, \ldots, x_{j+1}\right) \partial_{x_{j}}$ and that the function $\Theta$ given above is independent of $j$; otherwise the decomposition (6.1) would have been trivial. For any $1 \leq j \leq n$ denote by $\Delta^{j}=$ span $\left\{\partial_{x_{n-j+1}}, \ldots, \partial_{x_{n}}\right\}$ the module generated over the field of smooth functions, that is, each element of $\Delta^{j}$ is a linear combination of the vector fields $\partial_{x_{n-j+1}}, \ldots, \partial_{x_{n}}$ whose coefficients are smooth functions. We first verify easily that

$a d_{f} g=-\frac{\partial f_{n-1}}{\partial x_{n}} \partial_{x_{n-1}}-\frac{\partial f_{n}}{\partial x_{n}} \partial_{x_{n}}=\mu_{n-1}(x) \partial_{x_{n-1}}+\vartheta_{n-1}(x)$

where $\mu_{n-1}(x)=-\frac{\partial f_{n-1}}{\partial x_{n}}$ and $\vartheta_{n-1}(x) \in \Delta^{1}$. An induction argument implies that for any $1 \leq j \leq n-k-1$, we have

$$
a d_{f}^{j} g=\mu_{n-j}(x) \partial_{x_{n-j}}+\vartheta_{n-j}(x)
$$

where $\mu_{n-j}(x)=(-1)^{j} \prod_{i=1}^{j} \frac{\partial f_{n-i}}{\partial x_{n-i+1}}$ and $\vartheta_{n-j}(x) \in \Delta^{j}$. In particular for $j=n-k-1$ we have

$$
a d_{f}^{n-k-1} g=\mu_{k+1}(x) \partial_{x_{k+1}}+\vartheta_{k+1}(x)
$$


where $\vartheta_{k+1}(x) \in \Delta^{n-k-1}$. The Lie bracket with $f$ gives

$$
\begin{aligned}
a d_{f}^{n-k} g & =\sum_{j=1}^{k}\left[f_{j}\left(x_{1}, \ldots, x_{k+1}\right) \partial_{x_{j}}, \mu_{k+1} \partial_{x_{k+1}}+\vartheta_{k+1}\right] \\
& +\sum_{j=k+1}^{n}\left[f_{j}\left(x_{1}, \ldots, x_{j+1}\right) \partial_{x_{j}}, \mu_{k+1} \partial_{x_{k+1}}+\vartheta_{k+1}\right] \\
& =-\mu_{k+1}(x) \sum_{j=1}^{k} \frac{\partial f_{j}}{\partial x_{k+1}} \partial_{x_{j}}+\tilde{\vartheta}_{k},
\end{aligned}
$$

where $\tilde{\vartheta}_{k}(x) \in \Delta^{n-k}=\operatorname{span}\left\{\partial_{x_{k+1}}, \ldots, \partial_{x_{n}}\right\}$. This is due to the following facts:

(i) $a d_{f}^{n-k-1} g \in \Delta^{n-k}$;

(ii) $f_{j}\left(x_{1}, \ldots, x_{j+1}\right) \partial_{x_{j}} \in \Delta^{n-k}, \quad k+1 \leq j \leq n$;

(iii) $\left[f_{j}\left(x_{1}, \ldots, x_{k+1}\right) \partial_{x_{j}}, \Delta^{n-k}\right]=f_{j}(\cdot)\left[\partial_{x_{j}}, \Delta^{n-k}\right]$;

(iv) $\left[\Delta^{n-k}, \Delta^{n-k}\right] \subseteq \Delta^{n-k}$.

A simple calculation shows (using items (i)-(iv)) that

$$
\left[a d_{f}^{n-k} g, a d_{f}^{n-k-1} g\right]=\mu_{k+1}^{2}(x) \sum_{j=1}^{k} \frac{\partial^{2} f_{j}}{\partial x_{k+1}^{2}} \partial_{x_{j}}+\hat{\vartheta}_{k}(x)
$$

where $\hat{\vartheta}_{k} \in \Delta^{n-k}=\operatorname{span}\left\{\partial_{x_{k+1}}, \ldots, \partial_{x_{n}}\right\}$. The involutivity of $\mathcal{D}^{n-k+1}$ implies that

$$
\left[a d_{f}^{n-k} g, a d_{f}^{n-k-1} g\right]=\sum_{j=k}^{n} \delta_{n-j} a d_{f}^{n-j} g=\delta_{n-k} a d_{f}^{n-k} g+\breve{\vartheta}_{k}
$$

for some smooth functions $\delta_{0}, \delta_{1}, \ldots, \delta_{n-k}$. Comparing the two Lie brackets it follows that

$$
\left(\mu_{k+1}\right)^{2} \cdot \sum_{j=1}^{k} \frac{\partial^{2} f_{j}}{\partial x_{k+1}^{2}} \partial_{x_{j}}=-\left(\mu_{k+1}\right) \delta_{n-k} \cdot \sum_{j=1}^{k} \frac{\partial f_{j}}{\partial x_{k+1}} \partial_{x_{j}},
$$

that is, the condition

$$
\left(\mathcal{F} £_{k+1}\right) \triangleq \frac{\partial^{2} f_{j}}{\partial x_{k+1}^{2}}=\gamma_{n-k}(x) \frac{\partial f_{j}}{\partial x_{k+1}}, 1 \leq j \leq k .
$$

Notice that $\gamma_{n-k}=\gamma_{n-k}\left(x_{1}, \ldots, x_{k+1}\right)$ depends exclusively on the variables $x_{1}, \ldots, x_{k+1}$ since the components $f_{j}$ depend only on such variables. A double integration shows that there exist functions $F_{j}(x)$ and $\nu_{j}(x), 1 \leq j \leq k$ such that

$$
f_{j}\left(x_{1}, \ldots, x_{k+1}\right)=F_{j}\left(x_{1}, \ldots, x_{k}\right)+\nu_{j}\left(x_{1}, \ldots, x_{k}\right) \Theta(x)
$$

where

$$
\Theta(x)=\int_{0}^{x_{k+1}} \exp \left(\int_{0}^{t} \gamma_{n-k}\left(x_{1}, \ldots, x_{k}, \mathrm{~s}\right) \mathrm{ds}\right) \mathrm{dt}
$$

depends exclusively on $\gamma_{n-k}$ but not on the components. This achieves the proof of the claim.

\section{Proof of Theorem 2.2.}

Below we first give a brief proof of the constructive approach for rectifying nonsingular vector fields (Theorem 2.2) and we later address the convergence of the series.

Proof of Theorem 2.2 (i). Notice that for any diffeomorphism $z=\varphi(x)$ the two following conditions are equivalent.

(a) $\varphi_{*}(\nu)(z)=\partial_{z_{n}}$.

(b) $L_{\nu}\left(\varphi_{j}\right)(x)=0$ and $L_{\nu}\left(\varphi_{n}\right)(x)=1$ for $1 \leq j \leq n-1$.

For that reason we will show that condition (b) holds. To start let us take $1 \leq j \leq n-1$. It follows directly

$$
\begin{aligned}
L_{\nu}\left(\varphi_{j}\right)(x)= & L_{\nu}\left(x_{j}\right)+\sum_{s=1}^{\infty} L_{\nu}\left(\frac{(-1)^{s} x_{n}^{s}}{s !} L_{\sigma_{n} \nu}^{s-1}\left(\sigma_{n} \nu_{j}\right)\right) \\
= & \nu_{j}(x)+\sum_{s=1}^{\infty} \frac{(-1)^{s} x_{n}^{s}}{s !} L_{\nu} L_{\sigma_{n} \nu}^{s-1}\left(\sigma_{n} \nu_{j}\right) \\
& +\sum_{s=1}^{\infty} \frac{(-1)^{s} x_{n}^{s-1}}{(s-1) !} \nu_{n}(x) L_{\sigma_{n} \nu}^{s-1}\left(\sigma_{n} \nu_{j}\right) \\
= & \nu_{j}(x)+\sum_{s=1}^{\infty} \frac{(-1)^{s} x_{n}^{s}}{s !} \nu_{n}(x) L_{\sigma_{n} \nu}^{s}\left(\sigma_{n} \nu_{j}\right) \\
& -\nu_{j}(x)-\sum_{s=1}^{\infty} \frac{(-1)^{s} x_{n}^{s}}{s !} \nu_{n}(x) L_{\sigma_{n} \nu}^{s}\left(\sigma_{n} \nu_{j}\right)=0 .
\end{aligned}
$$

A direct computation shows that

$$
\begin{aligned}
L_{\nu} \varphi_{n}(x)= & \sum_{s=1}^{\infty} L_{\nu}\left(\frac{(-1)^{s-1} x_{n}^{s}}{s !} L_{\sigma_{n} \nu}^{s-1}\left(\sigma_{n}\right)\right) \\
= & \sum_{s=1}^{\infty} \frac{(-1)^{s-1} x_{n}^{s}}{s !} L_{\nu} L_{\sigma_{n} \nu}^{s-1}\left(\sigma_{n}\right) \\
& +\sum_{s=1}^{\infty} \frac{(-1)^{s-1} x_{n}^{s-1}}{(s-1) !} \nu_{n}(x) L_{\sigma_{n} \nu}^{s-1}\left(\sigma_{n}\right) \\
= & \sum_{s=1}^{\infty} \frac{(-1)^{s-1} x_{n}^{s}}{s !} \nu_{n}(x) L_{\sigma_{n} \nu}^{s}\left(\sigma_{n}\right) \\
& +\nu_{n}(x) \sigma_{n}(x)+\sum_{s=1}^{\infty} \frac{(-1)^{s} x_{n}^{s}}{s !} \nu_{n}(x) L_{\sigma_{n} \nu}^{s}\left(\sigma_{n}\right) \\
= & \nu_{n}(x) \sigma_{n}(x)=1 .
\end{aligned}
$$

This ends the sketch of proof of Theorem 2.2 (i).

Proof of Theorem 2.2 (ii). The proof of the inverse is constructive. It is enough to show it in the case $k=n$, that is, we suppose $\nu(0)=\partial_{z_{n}}$. The general case follows by first applying the following permutation

$$
\tau(x) \triangleq\left\{\begin{array}{l}
\tilde{x}_{j}=\tau_{j}(x)=x_{j}, \quad j \neq k, j \neq n \\
\tilde{x}_{k}=\tau_{k}(x)=x_{n} \\
\tilde{x}_{n}=\tau_{n}(x)=x_{k}
\end{array}\right.
$$

We look for a change of coordinates $x=\psi(z)$ that satisfies $\frac{\partial \psi(z)}{\partial z_{n}}=\nu(\psi(z))$. First, we extend $\nu$ in $\mathbb{R}^{n+1}$ as

$$
\hat{\nu}(x, y)=\hat{\nu}_{1}(x, y) \partial_{x_{1}}+\cdots+\hat{\nu}_{n}(x, y) \partial_{x_{n}}+\hat{\nu}_{n+1}(x, y) \partial_{y},
$$


where $\hat{\nu}_{j}=\nu_{j}(x)$ for $1 \leq j \leq n$, and $\hat{\nu}_{n+1}=\nu_{n}(x)$. We want emphasize here the fact that the components $\hat{\nu}_{n}(x, y)$ and $\hat{\nu}_{n+1}(x, y)$ are both equal to $\nu_{n}(x)$.

Because $\hat{\nu}(0) \neq 0$ there exist a change of coordinates $(z, w)=\hat{\varphi}(x, y)$ such that $\hat{\varphi}_{*} \hat{\nu}=\partial_{z_{n}}+\partial_{w}$. An inverse $(x, y)=\hat{\psi}(z, w)$ should thus satisfy

$$
\frac{\partial \hat{\psi}}{\partial z_{n}}+\frac{\partial \hat{\psi}}{\partial w}=\hat{\nu}(\hat{\psi}(z, w))
$$

Define the operator $\nabla \triangleq \partial_{z_{n}}+\partial_{w}$ and rewrite (6.2) as $\nabla \hat{\psi}=\hat{\nu}(\hat{\psi}(z, w))$. Apply the operator $\nabla$ again on both side and get (we put $\nabla^{2} \triangleq \nabla \circ \nabla$ )

$$
\begin{aligned}
\nabla^{2} \hat{\psi}(z, w) & =\nabla \hat{\nu}(\hat{\psi}(z, w)) \\
& =\frac{\partial \hat{\nu}}{\partial(x, y)}(\hat{\psi}(z, w)) \nabla \hat{\psi}(z, w) \\
& =\frac{\partial \hat{\nu}}{\partial(x, y)}(\hat{\psi}(z, w)) \hat{\nu}(\hat{\psi}(z, w)) \\
& =\left(L_{\hat{\nu}} \hat{\nu}\right)(\hat{\psi}(z, w)) .
\end{aligned}
$$

A simple recurrence argument yields

$$
\nabla^{s} \hat{\psi}(z, w)=\left(L_{\hat{\nu}}^{s-1} \hat{\nu}\right)(\hat{\psi}(z, w)), \text { for all } s \geq 1 .
$$

Define $\partial_{w}^{s} \triangleq \frac{\partial^{s}}{\partial w^{s}}$, and $\partial_{z_{n}}^{s} \triangleq \frac{\partial^{s}}{\partial z_{n}^{s}}$ for all $s \geq 1$. Since on the one hand side, $\partial_{w}=-\partial_{z_{n}}+\nabla$ and on the other hand side $\nabla \circ \partial_{z_{n}}=\partial_{z_{n}} \circ \nabla$, it follows that

$$
\partial_{w}^{s}=\sum_{i=0}^{s}(-1)^{i} C_{s}^{i} \partial_{z_{n}}^{i} \circ \nabla^{s-i}
$$

where $\partial_{z_{n}}^{s} \circ \nabla^{0}=\partial_{z_{n}}^{s}$ and $\partial_{z_{n}}^{0} \circ \nabla^{s}=\nabla^{s}$. We deduce that

$$
\begin{aligned}
\frac{\partial^{s} \hat{\psi}}{\partial w^{s}} & =\sum_{i=0}^{s}(-1)^{i} C_{s}^{i} \partial_{z_{n}}^{i}\left(\nabla^{s-i} \hat{\psi}(z, w)\right) \\
& =(-1)^{s} \frac{\partial^{s} \hat{\psi}(z, w)}{\partial z_{n}^{s}}+\sum_{i=0}^{s-1}(-1)^{i} C_{s}^{i} \partial_{z_{n}}^{i} L_{\hat{\nu}}^{s-i-1}(\hat{\nu})(\hat{\psi}(z, w))
\end{aligned}
$$

Taking $\hat{\psi}(z, 0)=(z, 0)$, we get

$$
\left.\frac{\partial^{s} \hat{\psi}}{\partial w^{s}}\right|_{w=0}=\sum_{i=0}^{s-1}(-1)^{i} C_{s}^{i} \partial_{z_{n}}^{i} L_{\hat{\nu}}^{s-i-1}(\hat{\nu})(z, 0) .
$$

A Taylor series expansion of $\hat{\psi}(z, w)$ with respect to $w$ at $w=0$ is

$\hat{\psi}(z, w)=\left(\begin{array}{c}z \\ 0\end{array}\right)+\sum_{s=1}^{\infty} \frac{w^{s}}{s !}\left(\sum_{i=0}^{s-1}(-1)^{i} C_{s}^{i} \partial_{z_{n}}^{i} L_{\hat{\nu}}^{s-i-1}(\hat{\nu})(z, 0)\right)$

Let us define $\psi(z)$ by its components in the following way: for any $1 \leq j \leq n$ we set $\psi_{j}(z)=\left.\hat{\psi}_{j}(z, w)\right|_{w=z_{n}}$. Since for any $1 \leq j \leq n, \hat{\nu}_{j}(x, y)=\nu_{j}(x)$ is independent of the variable $y$, it follows that $L_{\hat{\nu}}^{s} \hat{\nu}_{j}=L_{\nu}^{s} \nu_{j}$ for all $s \geq 0$. We then deduce that

$$
\psi_{j}(z)=z_{j}+\sum_{s=1}^{\infty} \frac{z_{n}^{s}}{s !}\left(\sum_{i=0}^{s-1}(-1)^{i} C_{s}^{i} \partial_{z_{n}}^{i} L_{\nu}^{s-i-1}\left(\nu_{j}\right)(z)\right) .
$$

To complete the proof we will show that $\frac{\partial \psi_{j}(z)}{\partial z_{n}}=\nu_{j}(\psi(z))$ for all $1 \leq j \leq n$; which indeed follows from the fact that

$$
\begin{aligned}
\frac{\partial \psi_{j}(z)}{\partial z_{n}}=\frac{\partial}{\partial z_{n}} \hat{\psi}_{j}\left(z, z_{n}\right) & =\frac{\partial \hat{\psi}_{j}}{\partial z_{n}}\left(z, z_{n}\right)+\frac{\partial \hat{\psi}_{j}}{\partial w}\left(z, z_{n}\right) \\
& =\hat{\nu}_{j}\left(\hat{\psi}\left(z, z_{n}\right)\right)=\nu_{j}(\psi(z)) .
\end{aligned}
$$

This ends the proof-sketch of Theorem 2.2.

Convergence. Let us first introduce some useful notation. For any $x \in \mathbb{R}^{n}$ we put $x=\left(x_{1}, \ldots, x_{n}\right)$. For the subset $\mathbb{N}^{n} \subset \mathbb{R}^{n}$ of $n$-tuples of integers we use a bolded variable to denote its elements. Given two $n$-tuples $\boldsymbol{m}=\left(m_{1}, \ldots, m_{n}\right)$ and $\boldsymbol{\alpha}=\left(\alpha_{1}, \ldots, \alpha_{n}\right)$ we say that $\boldsymbol{m} \succeq \boldsymbol{\alpha}$ if and only if $m_{i} \geq \alpha_{i}$ for all $1 \leq i \leq n$ and we denote by $\boldsymbol{m} !=m_{1} ! \cdots m_{n}$ ! and $\boldsymbol{m}^{\boldsymbol{\alpha}}=m_{1}^{\alpha_{1}} \cdots m_{n}^{\alpha_{n}}$. By extension, for $x=\left(x_{1}, \ldots, x_{n}\right) \in \mathbb{R}^{n}$ we put $\boldsymbol{x}^{\boldsymbol{m}}=$ $x_{1}^{m_{1}} \cdots x_{n}^{m_{n}}$ and put $|\boldsymbol{m}|=m_{1}+\cdots+m_{n}$. Let $f$ be an analytic function with $f(x)=\sum f_{\boldsymbol{m}} \cdot x^{\boldsymbol{m}}$ its Taylor series expansion where $f_{\boldsymbol{m}}=\frac{1}{\boldsymbol{m} !} \frac{\partial^{\boldsymbol{m}} f}{\partial x^{\boldsymbol{m}}}(0)$ are constant coefficients, and $\nu=\nu_{1} \partial_{x_{1}}+\cdots+\nu_{n} \partial_{x_{n}}$ an analytic vector field. For any $\rho>0$ we define the norm $\|\cdot\|_{\rho}$ by $\|f\|_{\rho}=\sum\left|f_{\boldsymbol{m}}\right| \cdot \rho^{|\boldsymbol{m}|}$ and extend the norm to vector fields by $\|\nu\|_{\rho}=\max \left\{\left\|\nu_{1}\right\|_{\rho}, \ldots,\left\|\nu_{n}\right\|_{\rho}\right\}$.

(i) We now prove the convergence of the series

$$
\varphi_{j}(x)=x_{j}+\sum_{s=1}^{\infty} \frac{(-1)^{s} x_{k}^{s}}{s !} L_{\sigma_{k} \nu}^{s-1}\left(\sigma_{k} \nu_{j}\right)(x) .
$$

Assume $\nu_{k}(0) \neq 0$ and put $\sigma_{k}=1 / \nu_{k}(x)$ and take $f=$ $\sigma_{k} \nu_{j}$. Choose $\rho>0$ such that $\left\|\sigma_{k} \nu_{j}\right\|_{\rho}=\kappa_{j}(\rho)<+\infty$ for all $1 \leq j \leq n$ and put $\kappa(\rho)=\max \left\{\kappa_{1}(\rho), \ldots, \kappa_{n}(\rho)\right\}$. Using Lemma 6.1 (ii) below we obtain, for any $0<\hat{\rho}<\rho$ and any $s \geq 1$, that

$$
\begin{aligned}
\left\|L_{\sigma_{k} \nu}^{s-1}(f)\right\|_{\hat{\rho}} & \leq(s-1) !(\hat{\rho} \ln (\rho / \hat{\rho}))^{-s+1}\|f\|_{\rho} \cdot(\kappa(\rho))^{s-1} \\
& \leq(s-1) !(\hat{\rho} \ln (\rho / \hat{\rho}))^{-s+1} \cdot(\kappa(\rho))^{s}
\end{aligned}
$$

Hence the norm of the series $\varphi_{j}(x)$ can be approximated by

$$
\begin{aligned}
\left\|\varphi_{j}(x)\right\|_{\hat{\rho}} & \leq \hat{\rho}+\sum_{s=1}^{\infty} \frac{\hat{\rho}^{s}}{s !}\left\|L_{\sigma_{k} \nu}^{s-1}(f)\right\|_{\hat{\rho}} \\
& \leq \hat{\rho}+\hat{\rho} \kappa(\rho) \cdot \sum_{s=1}^{\infty} \frac{1}{s}(\kappa(\rho) / \ln (\rho / \hat{\rho}))^{s-1} \\
& \leq \hat{\rho}+\hat{\rho} \kappa(\rho) \cdot \sum_{s=1}^{\infty}(\kappa(\rho) / \ln (\rho / \hat{\rho}))^{s-1}
\end{aligned}
$$

The series converges and is bounded by $\hat{\rho}+\frac{\hat{\rho} \kappa(\rho)}{1-\kappa(\rho) / \ln (\rho / \hat{\rho})}$ if $\kappa(\rho) / \ln (\rho / \hat{\rho})<1$, that is, if we choose $\hat{\rho}<\rho e^{-\kappa(\rho)}$.

(ii) To prove the convergence of the series

$$
\psi_{j}(z)=z_{j}+\sum_{s=1}^{\infty} \frac{z_{n}^{s}}{s !}\left(\sum_{i=0}^{s-1}(-1)^{i} C_{s}^{i} \partial_{z_{n}}^{i} L_{\nu}^{s-i-1}\left(\nu_{j}\right)(z)\right)
$$


we use Lemma 6.1 (iii). Taking $f=\nu_{j}$ we can estimate the component $\psi_{j}$ as follows

$$
\begin{aligned}
& \left\|\psi_{j}(z)\right\|_{\hat{\rho}} \leq \hat{\rho}+\sum_{s=1}^{\infty} \frac{\hat{\rho}^{s}}{s !}\left(\sum_{i=0}^{s-1} C_{s}^{i}\left\|\partial_{z_{n}}^{i} L_{\nu}^{s-i-1}\left(\nu_{j}\right)(z)\right\|_{\hat{\rho}}\right) \\
& \leq \hat{\rho}+\sum_{s=1}^{\infty} \frac{\hat{\rho}^{s}}{s !}\left(\sum_{i=0}^{s-1} C_{s}^{i}(s-1) !(\hat{\rho} \ln (\rho / \hat{\rho}))^{-s+1}\|f\|_{\rho}\|\nu\|_{\rho}^{s-i-1}\right) \\
& \leq \hat{\rho}+\hat{\rho}\|f\|_{\rho} \sum_{s=1}^{\infty} \frac{\ln (\rho / \hat{\rho})^{-s+1}}{s}\left(\sum_{i=0}^{s-1} C_{s}^{i} \kappa(\rho)^{s-i-1}\right) \\
& \leq \hat{\rho}+\hat{\rho}\|f\|_{\rho} \sum_{s=1}^{\infty} \frac{\ln (\rho / \hat{\rho})^{-s+1}}{s} \frac{(1+\kappa(\rho))^{s}-1}{\kappa(\rho)} \\
& \leq \hat{\rho}+\frac{\hat{\rho} \ln (\rho / \hat{\rho})}{\kappa(\rho)}\|f\|_{\rho} \sum_{s=1}^{\infty} \ln (\rho / \hat{\rho})^{-s}\left[(1+\kappa(\rho))^{s}-1\right]
\end{aligned}
$$

The series is convergent provided we chose $\frac{1+\kappa(\rho)}{\ln (\rho / \hat{\rho})}<1$, that is, whenever $\hat{\rho}<\rho e^{-1-\kappa(\rho)}$.

To complete the proof we need to establish Lemma 6.1 below. Before some more notation is needed. Let denote by $\partial_{i}: \mathcal{C}^{\omega}\left(\mathbb{R}^{n}\right) \longrightarrow \mathcal{C}^{\omega}\left(\mathbb{R}^{n}\right)$ the derivation operator with $\partial_{i}(f)=\frac{\partial f}{\partial x_{i}}$. For $\boldsymbol{\alpha}=\left(\alpha_{1}, \ldots, \alpha_{n}\right)$ we get

$$
\partial^{\boldsymbol{\alpha}}(f)=\partial_{1}^{\alpha_{1}} \circ \cdots \circ \partial_{n}^{\alpha_{n}}(f)=\frac{\partial^{\alpha} f}{\partial x^{\boldsymbol{\alpha}}}=\frac{\partial^{\alpha_{1}+\cdots+\alpha_{n}} f}{\partial x_{1}^{\alpha_{1}} \cdots \partial x_{n}^{\alpha_{n}}} .
$$

For the vector field $\nu: \partial^{\boldsymbol{\alpha}}(\nu)=\partial^{\boldsymbol{\alpha}}\left(\nu_{1}\right) \partial_{x_{1}}+\cdots+$ $\partial^{\boldsymbol{\alpha}}\left(\nu_{n}\right) \partial_{x_{n}}$. It is easy to see that

$$
L_{\nu}(f)=\sum_{j=1}^{n} \frac{\partial f}{\partial x_{j}} \nu_{j}=\sum_{j_{1}=1}^{n} \partial^{\boldsymbol{\alpha}_{0}}(f) \times \partial^{\boldsymbol{\alpha}_{1}}\left(\nu_{j_{1}}\right)
$$

where $\left|\boldsymbol{\alpha}_{\mathbf{0}}\right|=1$ and $\left|\boldsymbol{\alpha}_{\mathbf{1}}\right|=0$ with $\boldsymbol{\alpha}_{\mathbf{0}}$ an $n$-tuple whose components, except the $\left(j_{1}\right)^{t h}$ component are zero. By an inductive argument we check that for any $s \geq 1$ the successive Lie derivatives yield

$$
L_{\nu}^{s}(f)=\sum_{\mathcal{J}} \sum \partial^{\boldsymbol{\alpha}_{0}}(f) \partial^{\boldsymbol{\alpha}_{1}}\left(\nu_{j_{1}}\right) \cdots \partial^{\boldsymbol{\alpha}_{s-1}}\left(\nu_{j_{s-1}}\right) \partial^{\boldsymbol{\alpha}_{s}}\left(\nu_{j_{s}}\right),
$$

where $\mathcal{J}=\left\{j_{1}, \ldots, j_{s}, 1 \leq j_{i} \leq n\right\}$ and the second summation is taken over some $n$-tuples $\boldsymbol{\alpha}_{\boldsymbol{i}}=\left(\alpha_{i 1}, \ldots, \alpha_{i n}\right)$, $i=0,1, \ldots, s$ with $\boldsymbol{\alpha}_{\boldsymbol{s}}=\mathbf{0},\left|\boldsymbol{\alpha}_{\mathbf{0}}\right| \geq 1$ and $\left|\boldsymbol{\alpha}_{\mathbf{0}}\right|+\left|\boldsymbol{\alpha}_{\mathbf{1}}\right|+$ $\cdots+\left|\boldsymbol{\alpha}_{\boldsymbol{s}}\right|=s$. Let the Taylor expansions of the analytic functions $f, \nu_{j_{1}}, \ldots, \nu_{j_{s}}$ be represented by

$$
f(x)=\sum_{\boldsymbol{m}_{\mathbf{0}} \succeq 0} f_{\boldsymbol{m}_{\mathbf{0}}} x^{\boldsymbol{m}_{\mathbf{0}}} \text { and } \nu_{j_{i}}(x)=\sum_{\boldsymbol{m}_{\boldsymbol{i}} \succeq 0}\left(\nu_{j_{i}}\right)_{\boldsymbol{m}_{\boldsymbol{i}}} x^{\boldsymbol{m}_{\boldsymbol{i}}}
$$

for all $1 \leq i \leq s$. It follows easily that

$$
\partial^{\boldsymbol{\alpha}_{0}}(f)=\sum_{\boldsymbol{m}_{\mathbf{0}} \succeq \boldsymbol{\alpha}_{0}}\left(\boldsymbol{m}_{\mathbf{0}} ! / \boldsymbol{\alpha}_{\mathbf{0}} !\right) f_{\boldsymbol{m}_{\mathbf{0}}} x^{\boldsymbol{m}_{\mathbf{0}}-\boldsymbol{\alpha}_{0}},
$$

and for any $1 \leq i \leq s$

$$
\partial^{\boldsymbol{\alpha}_{i}}\left(\nu_{j_{i}}\right)=\sum_{\boldsymbol{m}_{\boldsymbol{i}} \succeq \boldsymbol{\alpha}_{\boldsymbol{i}}}\left(\boldsymbol{m}_{\boldsymbol{i}} ! / \boldsymbol{\alpha}_{\boldsymbol{i}} !\right)\left(\nu_{j_{i}}\right)_{\boldsymbol{m}_{\boldsymbol{i}}} x^{\boldsymbol{m}_{\boldsymbol{i}}-\boldsymbol{\alpha}_{\boldsymbol{i}}} .
$$

Consequently

$$
\begin{aligned}
& L_{\nu}^{s}(f)=\sum_{\mathcal{J}|\boldsymbol{\alpha}|=s} \sum_{\boldsymbol{m}_{\boldsymbol{i}} \succeq \boldsymbol{\alpha}_{\boldsymbol{i}}}\left[f_{\boldsymbol{m}_{\mathbf{0}}} \times\left(\nu_{j_{1}}\right)_{\boldsymbol{m}_{\mathbf{1}}} \times \cdots \times\left(\nu_{j_{s}}\right)_{\boldsymbol{m}_{\boldsymbol{s}}} \times\right. \\
& \left.\qquad\left(\boldsymbol{m}_{\mathbf{0}} ! / \boldsymbol{\alpha}_{\mathbf{0}} !\right) \cdots\left(\boldsymbol{m}_{\boldsymbol{s}} ! / \boldsymbol{\alpha}_{\boldsymbol{s}} !\right)\right] x^{\mathbf{m}-\boldsymbol{\alpha}}, \\
& \text { where, we put } \mathbf{m}=\boldsymbol{m}_{\mathbf{0}}+\cdots+\boldsymbol{m}_{\boldsymbol{s}} \text { and } \boldsymbol{\alpha}=\boldsymbol{\alpha}_{\mathbf{0}}+\cdots+\boldsymbol{\alpha}_{\boldsymbol{s}}
\end{aligned}
$$
for convenience of notation.

Lemma 6.1 Let $f$ (resp. $\nu$ ) be an analytic function (resp. vector field). Let $s \geq 1$ and $t \geq 0$ be given integers and $0<\hat{\rho}<\rho$ two positive real numbers. Define

$$
M=\sup _{\boldsymbol{m}_{\boldsymbol{i}} \succeq \boldsymbol{\alpha}_{i}}\left\{\sum_{|\boldsymbol{\alpha}|=s}\left(\boldsymbol{m}_{\mathbf{0}} ! / \boldsymbol{\alpha}_{\mathbf{0}} !\right) \cdots\left(\boldsymbol{m}_{\boldsymbol{s}} ! / \boldsymbol{\alpha}_{\boldsymbol{s}} !\right)(\hat{\rho} / \rho)^{|\mathbf{m}|}\right\} .
$$

Then we have the following inequalities

$$
\begin{array}{ll}
\text { (i) } & M \leq s !(\ln (\rho / \hat{\rho}))^{-s} \\
\text { (ii) } & \left\|L_{\nu}^{s}(f)\right\|_{\hat{\rho}} \leq s !(\hat{\rho} \ln (\rho / \hat{\rho}))^{-s}\|f\|_{\rho}\|\nu\|_{\rho}^{s} \\
\text { (iii) }\left\|\partial_{z_{n}}^{i} L_{\nu}^{t-i}(f)\right\| \hat{\rho} \leq t !(\hat{\rho} \ln (\rho / \hat{\rho}))^{-t}\|f\|_{\rho}\|\nu\|_{\rho}^{t-i}
\end{array}
$$

Proof of Lemma 6.1 (i) Because $m_{\boldsymbol{i}} ! / \alpha_{\boldsymbol{i}} ! \leq\left(m_{\boldsymbol{i}}\right)^{\alpha_{\boldsymbol{i}}}$ for all $\mathbf{0} \leq \boldsymbol{i} \leq \boldsymbol{s}$ we deduce that

$$
M \leq \sup _{\boldsymbol{m}_{\boldsymbol{i}} \succeq \boldsymbol{\alpha}_{\boldsymbol{i}}}\left\{\sum_{|\boldsymbol{\alpha}|=s}\left(\boldsymbol{m}_{\mathbf{0}}\right)^{\boldsymbol{\alpha}_{\mathbf{0}}} \cdots\left(\boldsymbol{m}_{\boldsymbol{s}}\right)^{\boldsymbol{\alpha}_{\boldsymbol{s}}}(\hat{\rho} / \rho)^{|\mathbf{m}|}\right\} .
$$

On the other side,

$$
\sum_{|\boldsymbol{\alpha}|=s}\left(\boldsymbol{m}_{\mathbf{0}}\right)^{\boldsymbol{\alpha}_{0}} \cdots\left(\boldsymbol{m}_{\boldsymbol{s}}\right)^{\boldsymbol{\alpha}_{s}} \leq\left(\left|\boldsymbol{m}_{\mathbf{0}}\right|+\cdots+\left|\boldsymbol{m}_{\boldsymbol{s}}\right|\right)^{s}=\mathbf{m}^{s}
$$

which implies that

$$
\begin{aligned}
M & \leq \sup _{\boldsymbol{m}_{\boldsymbol{i}} \succeq \boldsymbol{\alpha}_{\boldsymbol{i}}}\left\{\left(\left|\boldsymbol{m}_{\mathbf{0}}\right|+\cdots+\left|\boldsymbol{m}_{\boldsymbol{s}}\right|\right)^{s}(\hat{\rho} / \rho)^{|\mathbf{m}|}\right\} \\
& \leq \sup _{\boldsymbol{m}_{\boldsymbol{i}} \succeq \mathbf{0}}\left\{\left(\left|\boldsymbol{m}_{\mathbf{0}}\right|+\cdots+\left|\boldsymbol{m}_{\boldsymbol{s}}\right|\right)^{s}(\hat{\rho} / \rho)^{|\mathbf{m}|}\right\}
\end{aligned}
$$

The inequality follows from Stirling $s !=\sqrt{2 \pi s}(s / e)^{s} e^{\lambda_{s}}$ where $\lambda_{s}>0$, and the fact that the maximum of $x^{s}(\hat{\rho} / \rho)^{x}$ is $\left(\frac{s}{\ln (\rho / \hat{\rho})}\right)^{s} e^{-s}$. 
(ii) For any $0<\hat{\rho}<\rho$ we have the following estimates

$$
\begin{array}{r}
|| L_{\nu}^{s}(f)||_{\hat{\rho}}=\sum_{\mathcal{J}} \sum_{|\boldsymbol{\alpha}|=s} \sum_{\boldsymbol{m}_{\boldsymbol{i}} \geq \boldsymbol{\alpha}_{\boldsymbol{i}}}\left[\left|f_{\boldsymbol{m}_{\mathbf{0}}}\right|\left|\left(\nu_{j_{1}}\right)_{\boldsymbol{m}_{\mathbf{1}}}\right| \cdots\left|\left(\nu_{j_{s}}\right)_{\boldsymbol{m}_{\boldsymbol{s}}}\right|\right. \\
\left.\left(\boldsymbol{m}_{\mathbf{0}} ! / \boldsymbol{\alpha}_{\mathbf{0}} !\right) \cdots\left(\boldsymbol{m}_{\boldsymbol{s}} ! / \boldsymbol{\alpha}_{\boldsymbol{s}} !\right)\right] \hat{\rho}^{|\mathbf{m}-\boldsymbol{\alpha}|} \\
=\hat{\rho}^{-|\boldsymbol{\alpha}|} \sum_{\mathcal{J}} \sum_{|\boldsymbol{\alpha}|=s} \sum_{\boldsymbol{m}_{\boldsymbol{i}} \geq \boldsymbol{\alpha}_{\boldsymbol{i}}}\left[\left|f_{\boldsymbol{m}_{\mathbf{0}}}\right| \times\left|\left(\nu_{j_{1}}\right)_{\boldsymbol{m}_{\mathbf{1}}}\right| \times \cdots \times\left|\left(\nu_{j_{s}}\right)_{\boldsymbol{m}_{\boldsymbol{s}} \mid \times}\right| \times\right. \\
\left.\left(\boldsymbol{m}_{\mathbf{0}} ! / \boldsymbol{\alpha}_{\mathbf{0}} !\right) \cdots\left(\boldsymbol{m}_{\boldsymbol{s}} ! / \boldsymbol{\alpha}_{\boldsymbol{s}} !\right)\right] \rho^{|\mathbf{m}|}(\hat{\rho} / \rho)^{|\mathbf{m}|}
\end{array}
$$

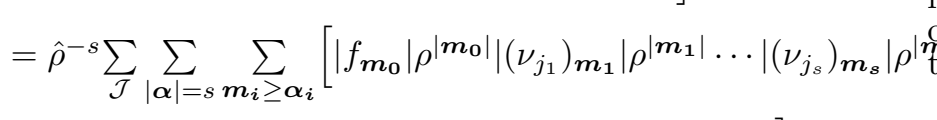$$
\left.\left(\boldsymbol{m}_{\mathbf{0}} ! / \boldsymbol{\alpha}_{\mathbf{0}} !\right) \cdots\left(\boldsymbol{m}_{\boldsymbol{s}} ! / \boldsymbol{\alpha}_{\boldsymbol{s}} !\right)\right](\hat{\rho} / \rho)^{|\mathbf{m}|}
$$$$
\leq \hat{\rho}^{-s} \sum_{\mathcal{J}} \sum_{|\boldsymbol{\alpha}|=s} \sum_{\boldsymbol{m}_{\boldsymbol{i}} \geq \boldsymbol{\alpha}_{\boldsymbol{i}}}\left[\left|f_{\boldsymbol{m}_{\mathbf{0}}}\right| \rho^{\left|\boldsymbol{m}_{\mathbf{0}}\right|}\left|\left(\nu_{j_{1}}\right)_{\boldsymbol{m}_{\mathbf{1}}}\right| \rho^{\left|\boldsymbol{m}_{\mathbf{1}}\right|} \ldots\left|\left(\nu_{j_{s}}\right)_{\boldsymbol{m}_{\boldsymbol{s}}}\right| \rho^{\mid \boldsymbol{m}_{\boldsymbol{s}}}\right.
$$$$
\left.\left(\boldsymbol{m}_{\mathbf{0}} ! / \boldsymbol{\alpha}_{\mathbf{0}} !\right) \mid \cdots\left(\boldsymbol{m}_{\boldsymbol{s}} ! / \boldsymbol{\alpha}_{\boldsymbol{s}} !\right)\right](\hat{\rho} / \rho)^{|\mathbf{m}|}
$$

$\leq \hat{\rho}^{-s}\|f\|_{\rho}\|\nu\|_{\rho}^{s} \sup _{\boldsymbol{m}_{\boldsymbol{i}} \succeq \boldsymbol{\alpha}_{\boldsymbol{i}}}\left\{\sum_{|\boldsymbol{\alpha}|=s}\left(\boldsymbol{m}_{\mathbf{0}} ! / \boldsymbol{\alpha}_{\mathbf{0}} !\right) \cdots\left(\boldsymbol{m}_{\boldsymbol{s}} ! / \boldsymbol{\alpha}_{\boldsymbol{s}} !\right)(\hat{\rho} / \rho)^{\left|\mathbf{m}_{\mid}\right|}\right\}^{[2]}$

Using item (i) above it follows that

$$
\left\|L_{\nu}^{s}(f)\right\|_{\hat{\rho}} \leq s !(\hat{\rho} \ln (\rho / \hat{\rho}))^{-s}\|f\|_{\rho}\|\nu\|_{\rho}^{s}
$$

Formula (6.3) follows directly if we replace $s$ by $s-1$, the vector field $\nu$ by $\sigma_{k} \nu$ and the function $f$ by $\sigma_{k} \nu_{j}$ taking into account that $\|f\|_{\rho} \leq \kappa(\rho)$.

(iii) Consider (6.4) where $s$ is replaced by $t-i$, that is,

$$
L_{\nu}^{t-i}(f)=\sum_{\mathcal{J}} \sum \partial^{\boldsymbol{\alpha}_{0}}(f) \partial^{\boldsymbol{\alpha}_{\mathbf{1}}}\left(\nu_{j_{1}}\right) \cdots \partial^{\boldsymbol{\alpha}_{t-i}}\left(\nu_{j_{t-i}}\right)
$$

with $\boldsymbol{\alpha}_{\boldsymbol{t}-i}=\mathbf{0},\left|\boldsymbol{\alpha}_{\mathbf{0}}\right| \geq 1$ and

$$
\left|\boldsymbol{\alpha}_{\mathbf{0}}\right|+\left|\boldsymbol{\alpha}_{\mathbf{1}}\right|+\cdots+\left|\boldsymbol{\alpha}_{\boldsymbol{t}-i}\right|=t-i .
$$

Differentiating $i$ times with respect to $x_{n}$ we get

$\partial_{x_{n}}^{i} L_{\nu}^{t-i}(f)=\sum_{\mathcal{J}} \sum \partial^{\hat{\boldsymbol{\alpha}}_{\mathbf{0}}}(f) \partial^{\hat{\boldsymbol{\alpha}}_{\mathbf{1}}}\left(\nu_{j_{1}}\right) \cdots \partial^{\hat{\boldsymbol{\alpha}}_{\boldsymbol{t - i}}}\left(\nu_{j_{t-i}}\right)$

with $\left|\hat{\boldsymbol{\alpha}}_{\mathbf{0}}\right| \geq 1$ and $\left|\hat{\boldsymbol{\alpha}}_{\mathbf{0}}\right|+\left|\hat{\boldsymbol{\alpha}}_{\mathbf{1}}\right|+\cdots+\left|\hat{\boldsymbol{\alpha}}_{\boldsymbol{t}-i}\right|=t$. Following the same steps in Lemma 6.1 (ii) we get $\left\|\partial_{x_{n}}^{i} L_{\nu}^{t-i}(f)\right\|_{\hat{\rho}} \leq t !(\hat{\rho} \ln (\rho / \hat{\rho}))^{-t}\|f\|_{\rho}\|\nu\|_{\rho}^{t-i}$. Notice that the power $t-i$ on the last term is due to the fact there are $t-i$ factors only that involve the components of the vector field $\nu$.

\section{Conclusion}

In this paper we provided algorithms allowing to compute (feedback) linearizing coordinates for single-input control systems. The algorithms are based on a successive rectification of one vector field at a time using explicit convergent power series of functions. The algorithms do not require an a priori checking of the (feedback) linearization conditions of Theorem 1.1 (which are usually very

\section{References}

1] J. Alvarez-Gallegos, Nonlinear regulation of a Lorenz system by feedback linearization techniques, Dynamics \& Control, (1994) 277-298.

[2] P. J. Antsaklis and A. N. Michel, Linear Systems, McGraw-Hill, (1997).

-A. Banaszuk, J. Hauser, Approximate feedback linearization: A homotopy operator approach, Proceedings of the American Control Conference, Baltimore, Maryland (1994), pp. 1690-1694.

hard). Indeed, at each step those conditions are replaced with the fact that the second derivative of a certain vecfield is zero (state linearization) or proportional to its (fespect to same variable (feed case). Thus at each step, the previous system is transaffine lower dimensional system and a linear one (or feedback form) whose first variable acts as control input for the lower system. The extension of our results to the multicase is in progress. We expect to apply the explicit finding coordinates that simultaneously rectify a given set f vector fields. The algorithms will then be generalized R. W. Brockett, Feedback invariants for nonlinear systems, in Proc. IFAC Congress, Helsinski, 1978.

[5] G. O. Guardabassi, S. M. Savaresi, Approximate linearization via feedback-an overview, Automatica vol 37(2001) pp. 1-15.

[6] K. Guemghar, B. Srinivasan, D. Bonvin, Approximate inputoutput linearization of nonlinear systems using the observability normal form, European Conference Control (2003).

[7] Q. Gong, W. Kang and I. M. Ross, A pseudospectral method for the optimial control of constrained feedback linearizable systems, IEEE Trans. Autom. Contr., 51(2006), pp. 1115-1129.

[8] L. Guzzella, A. Isidori, On approximate linearization of nonlinear control systems, International Journal of Robust and Nonlinear Control vol 3(3) pp. 261-276.

[9] J. Hauser, S. Sastry, P. Kokotovic, Nonlinear control via approximate input-output linearization: The ball and beam example, IEEE Trans. Autom. Contr., 37:3(1992), pp. 392-398.

[10] M-T. Ho, Y-W. Tu, and H-S. Lin, Controlling a Ball and Wheel System using Full-State-Feedback Linearization: A Testbed for Nonlinear Control Design, in IEEE Control Systems magazine, October 2009, vol. 29(5) pp. 93-101.

[11] L. R. Hunt and R. Su, Linear equivalents of nonlinear time varying systems, in Proceedings of Mathematical Theory of Networks \& Systems, Santa Monica, CA, USA, (1981), pp. 119-123.

[12] A. Isidori, Nonlinear Control Systems, 3rd ed., Springer, London, 1995.

[13] B. Jakubczyk and W. Respondek, On linearization of control systems, Bull. Acad. Polon. Sci. Ser. Math., 28, (1980), pp. 517522 .

[14] T. Kailath, Linear Systems, Prentice Hall Information and System Sciences Series, USA, (1980).

[15] W. Kang, Approximate linearization of nonlinear control systems, Systems \& Control Letters Vol 23:1(1994), pp. 43-52.

[16] A. J. Krener, On the equivalence of control systems and the linearization of nonlinear systems, SIAM Journal on Control, 11 (1973) pp. 670-676.

[17] A. J. Krener, Approximate linearization by state feedback and coordinate change, Systems \& Control Letters, vol 5(3), 1984, pp. 181-185.

[18] M. Krstic, Feedback linearizability and explicit integrator forwarding controllers for classes of feedforward systems, in IEEE Trans. Automat. Control, 49, (2004), pp. 1668-1682.

[19] M. Krstic, Explicit Forwarding Controllers-Beyond Linearizable Class, in Proceedings of the 2005 American Control Conference, Portland, Oregon, USA, (2005), pp. 3556-3561. 
[20] M. Krstic, Feedforward systems linearizable by coordinate change, in Proceedings of the 2004 American Control Conference, Boston, Massachussetts, USA, (2004), pp. 4348-4353.

[21] D. A. Lawrence, W. J. Rugh, Input-output pseudolinearization for nonlinear systems, IEEE Trans. Autom. Contr., vol 39:11(1994), pp. 2207-2218.

[22] C. Liqun and L. Yanzhu, Control of the Lorenz chaos by the exact linearization, Applied Mathematics \& Mechanics, 19(1998), pp. $67-73$

[23] A. S. Morse, Structural Invariants of Linear Multivariable Systems, SIAM Journal of Control 11(3), (1973), pp. 446-465.

[24] Ph. Mullhaupt, Quotient submanifolds for static feedback linearization in Systems \& Control Letters, 55(2006), pp. 549-557.

[25] H. Nijmeijer and A. J. van der Schaft, Nonlinear Dynamical Control Systems Springer-Verlag, New York, (1990).

[26] M. Schlemmer and S. K. Agrawal, Globally feedback linearizable time-invariant systems: optimal solution for Mayer's problem, $J$. of Dynam. Syst., Measurem. \& Contr. 122:2(2000), pp. 343-347.

[27] A. Serrani, A. Isidori, C. I. Byrnes, and L. Marconi, Recent advances in output regulation of nonlinear systems, Nonlinear Control in the Year 2000, A. Isidori, F. Lamnabhi-Lagarrigue and W. Respondek (eds), LNCIS vol. 259, 2(2000), pp. 409-419.

[28] I.A. Tall and W. Respondek, On Linearizability of Strict Feedforward Systems, in Proceedings of the 2008 American Control Conference, Seattle, Washington, USA, pp. 1929-1934.

[29] I.A. Tall and W. Respondek, Feedback Linearizable Strict Feedforward Systems, in Proceedings of the $4 \%$ th IEEE Conference on Decision and Control, (2008), Cancún, Mexico, pp. 2499-2504.

[30] I.A. Tall, Linearizable Feedforward Systems: A Special Class, in Proceedings of the 1\%th IEEE Conference on Control and Applications Multi-conference on Systems and Control, San Antonio, TX (2008), pp. 1201-1206.

[31] I.A. Tall, (Feedback) Linearizable Feedforward Systems: A Special Class, to appear in IEEE Trans. Autom. Contr..

[32] I.A. Tall, Flow Box Theorem and Beyond, to be submitted.

[33] I.A. Tall, State Linearization of Nonlinear Control Systems: An Explicit Algorithm 48th IEEE Conference on Decison and Control, China, pp. 7448-7453.

[34] I.A. Tall, Explicit Feedback Linearization of Nonlinear Control Systems, in 48th IEEE Conference on Decison and Control, China, pp. 7454-7459.

[35] I.A. Tall and W. Respondek, Analytic Normal Forms and Symmetries of Strict Feedforward Control Systems, to appear in International Journal of Robust and Nonlinear Control.

[36] S. Talwar, N. Sri Namachchivaya, and P. G. Voulgaris, Approximate Feedback Linearization: A Normal Form Approach, J. Dyn. Sys., Meas., Control, 118:2(1996), pp. 201-210. 